\title{
G (2) \\ Modeling and Analyzing of Nonlinear Dynamics for Linear Guide Slide Platform Considering Assembly Error
}

\section{Zhendong Liu}

northeastern university

Mengtao Xu

northeastern university

Hongzhuang Zhang

northeastern university

Changyou Li ( $\boldsymbol{\sigma}$ chyli@mail.neu.edu.cn )

Northeastern University https://orcid.org/0000-0003-0076-074X

Guo Yao

northeastern university

Huihui Miao

Northeastern University

Chenyu Wang

Northeastern University

Yimin Zhang

Zhaoging University

\section{Research Article}

Keywords: Linear guideway, Assembly error, Nonlinear dynamics, Dynamics modeling

Posted Date: August 23rd, 2021

Dol: https://doi.org/10.21203/rs.3.rs-817365/v1

License: @ (1) This work is licensed under a Creative Commons Attribution 4.0 International License.

Read Full License

Version of Record: A version of this preprint was published at Nonlinear Dynamics on March 24th, 2022. See the published version at https://doi.org/10.1007/s11071-022-07345-2. 
Modeling and analyzing of nonlinear dynamics for linear guide slide platform considering assembly error

\author{
Zhendong Liu ${ }^{\mathrm{a}}$, Mengtao Xu $\mathrm{X}^{\mathrm{a}}$, Hongzhuang Zhang ${ }^{\mathrm{a}}$, Changyou $\mathrm{Li}^{* a}$,Guo Yao ${ }^{* a}$, \\ Zhenyuan $\mathrm{Li}^{\mathrm{a}}$, HuiHui Miao ${ }^{\mathrm{a}}$, Chenyu Wang ${ }^{\mathrm{a}}$, Yimin Zhang ${ }^{\mathrm{b}}$ \\ ${ }^{a}$ School of Mechanical Engineering and Automation, Northeastern University, \\ Shenyang, 110819, China \\ ${ }^{\mathrm{b}}$ College of Mechanical and Automotive Engineering, Zhaoqing University, Zhaoqing \\ 526061, China
}

\begin{abstract}
:
In this study, a novel five degrees-of-freedom nonlinear dynamic model of linear guide slide platform is proposed, the model considers the effect of assembly error. The assembly errors are modeled as five types including: straightness assembly errors along $x$ and $y$ axes and rotation assembly error around $x, y$ and $z$ axes. The assembly error induced displacement of platform is considered. The restoring force and moment of the carriages is equal to zero when no dynamic or static load applied on the platform with considering the influence of assembly error. The influence of assembly error on the static and dynamic characteristics are investigated. The results indicate that the assembly error can cause uneven load distribution, change the dynamics of the system. In addition, the stability of the system cannot be improved by simply increasing the preload. Moreover, a series of experiments are conducted on a specialized platform to estimate the parameters of the system and verify the proposed model.
\end{abstract}

Keywords: Linear guideway; Assembly error; Nonlinear dynamics; Dynamics modeling

\title{
1. Introduction
}

Due to low friction, high stiffness, long service life, high precision, excellent dynamic response, run smoothly and easy to install, the linear guide can be used in machine tools with high vibration requirement such as grinding machine[1-3]. In machine tools, three most significant errors are geometric error, thermal error and cutting-force induced error [4]. The human error and routine maintenance induced geometric error can lead unequal contact deformation of each ball, cause groove wear, and then reduce the service life of linear guide carriage. In addition, the dynamic characteristics can be influenced by the variation of system parameters. Therefore, in order to have a deeper understanding of the influence of geometric errors on the dynamic and static characteristics of linear guide slide platform, it is necessary to proposed a more comprehensive analytical model.

Lots of researches focus on the static and dynamic characteristics of linear guideway under variety of conditions. To accurately investigate the dynamic characteristics of linear guide, Kong et al. [5] proposed a piecewise-nonlinear model, studied the simulation results based on the multi-term incremental harmonic balance method, it revealed that higher order nonlinearities was more responsive to the dynamic behavior than lower-order nonlinearities. Parivash et al. [6] proposed a coupled tribology-dynamic model of linear guideways with consideration of the influence of the lubricant and the coupling between vertical degrees and horizontal directions, the results showed that it was necessary to consider the coupled solution of vertical and horizontal directions into the lubricated contacts. Pawełko et al. [7] proposed a new methodology and method to model the preloaded rolling guides, and various degrees of simplification of the modeling methods were studied. Yang et al. [8] developed a hybrid

\footnotetext{
* Corresponding authors.

Email addresses: chyli@mail.neu.edu.cn (C. Li), yaoguo@me.neu.edu.cn(G.Yao)
} 
model of linear rolling guideway with considering the flexibility of the block, and the complex loading conditions in the vertical and horizontal directions are considered. Yasunori and Tanakab [9] studied the relationship between nonlinear friction and vibration of linear rolling guideway, and the influence of lubricant specifications on the damping ratio was investigated. Li et al. [10] proposed a time-varying model with consideration of crowing of rolling linear guide, and the ball passing frequency was predicted. In addition, the proposed dynamic model was validated by an experiment. Wang et al. [11] developed a three degrees of freedom nonlinear dynamic model to investigate the vibration characteristic of machine tool platform mounted on linear guideways, and the mechanics and contact characteristic were considered in the model. Wang et al. [12] investigated the relationship between vibration and groove wear of linear guideways, and analyzed the failure mechanism of rolling linear guide. Ohta and Tanaka [13] developed a static model of linear guideway with consideration of the flexibility of the carriage and rail, and the calculated vertical stiffness obtained from the theoretical formulations were compared with the measured vertical results. Hung et al. [14] developed a finite element model to studied the dynamic behaviors and cutting stability of a vertical milling system. The model used Hertzian contact theory for coupling the linear components and the machine structures. To reduce the ball passage vibration of a linear guideway, Ohta et al. [15] proposed a method of designing crowning, the method used approximate curve to control point. Sun et al. [16] presented a 5-degrees-of-freedom static model of linear roller bearing, the structure deformation of the carriage was calculated by finite element method, and the developed model was verified by comparing with the calculation results obtained by commercial software. Sun et al. [17] proposed an analytical model using hertz contact theory, on the base of which a full finite element model was developed. Moreover, the influence of external load and preload on the static characteristics were analyzed. Considering the contact stiffness of the interface between ball and groove, Lin et al. [18] proposed a finite element model to study the dynamic stiffness of the spindle head. The results from simulation and experimental showed that the dynamic stiffness increased with the preload of the linear guide. Zou and Wang [19] proposed a contact stiffness model which took wear and thermal deformation of linear guideway into account, and an experiment on a specialized linear guide was conducted to validate the model. Ohta [20] investigated the relationship between sound pressure level and linear velocity, and found that the main frequency of vibration response was caused by the rolling vibration, pitching vibration and vertical vibration of the carriage. Tao et al. [21] proposed a calculation model of stiffness of carriage based on Archard wear theory, the amount of wear of the grooves of linear guideway was predicted, and a series of experiments were conducted to verified the simulation results.

The above mentioned studies focused on the modeling and understanding the dynamic behaviors with different system parameters. Besides, the modeling and analysis of geometric error of mechanical systems had been reported by researchers. Liu et al. [22] using homogeneous coordinate transformation to model the position deviation of machine tool table system, and the dynamic response of the system was obtained by the integration of Newmark and Monte Carlo method. Pawel [23] proposed a new method to model the geometric deviations of machine tool table, and the influence of the geometric errors on joint kinematic errors was discussed. Ma et al. [24] developed an analytical model with consideration of the assembly errors of linear axis of CNC machine tool, the straightness and rotation deviations were calculated under four different conditions. Sun et al. [25] proposed a model to establish the relationship between assembly error and positioning accuracy, and the influence of volumetric 
errors of linear guideways on repeatability of positioning accuracy based on genetic algorithm was studied. To enhance the assembly performance of machine center, Sun [26] proposed a method based on computer aid engineering analysis. Mayer and Cloutier [27] proposed an analytical model and conducted a series of experiments to study the relations between the geometric error of linear guideway and the motion error of carriage.

Concluding from the studies, previous researches concerning the modeling of linear guide and discussing the influence of variety of parameters, few studies propose a comprehensive model and give the full consideration to every possible assembly error, which has a significant influence on positioning accuracy. The outline of this paper is as follows: after the introduction in section 1, section 2 modeled five types of assembly errors, and proposed a 5-degrees-of-freedom dynamic model of linear guide slide platform. Section 3 revealed the combined influence of assembly error and system parameters on the dynamic and static characteristic of the system. In addition, a series of experiments had been conducted to validate the proposed model and estimate the parameter of system.

\begin{tabular}{|c|c|}
\hline $\begin{array}{l}\text { Nomenclature } \\
\Delta \delta_{x}^{\varepsilon}, \Delta \delta_{y}^{\varepsilon}\end{array}$ & Straightness assembly errors of rail II in $x$ and $y$ directions \\
\hline$\Delta \varepsilon_{x}, \Delta \varepsilon_{y}, \Delta \varepsilon_{z}$ & Rotation assembly errors of rail II about $x, y$ and $z$ axis \\
\hline$\Delta x_{i j k}^{a}, \Delta y_{i j k}^{a}$ & $\begin{array}{l}\text { Type } a \text { assembly error induced contact deformation along } x \text { and } y \\
\text { axis }\end{array}$ \\
\hline$\Delta x_{i j k}^{b}, \quad \Delta y_{i j k}^{b}$ & $\begin{array}{l}\text { Type } b \text { assembly error induced contact deformation along } x \text { and } y \\
\text { axis }\end{array}$ \\
\hline$\Delta x_{i j k}^{c}, \Delta y_{i j k}^{c}$ & $\begin{array}{l}\text { Type } c \text { assembly error induced contact deformation along } x \text { and } y \\
\text { axis }\end{array}$ \\
\hline$\Delta x_{i j k}^{d}, \quad \Delta y_{i j k}^{d}$ & $\begin{array}{l}\text { Type } d \text { assembly error induced contact deformation along } x \text { and } y \\
\text { axis }\end{array}$ \\
\hline$\Delta x_{i j k}^{e}, \Delta y_{i j k}^{e}$ & $\begin{array}{l}\text { Type } e \text { assembly error induced contact deformation along } x \text { and } y \\
\text { axis }\end{array}$ \\
\hline$l_{k}$ & $\begin{array}{l}\text { distance between the } k^{\text {th }} \text { and the first loaded ball in the grooves of } \\
\text { each carriage }\end{array}$ \\
\hline$S_{0}^{\varepsilon}$ & $\begin{array}{l}\text { initial distance between the groove curvature centers with } \\
\text { consideration of assembly error }\end{array}$ \\
\hline$r_{\mathrm{cg}}, r_{\mathrm{rg}}$ & the groove radius of carriage and rail \\
\hline$\alpha_{0}^{\varepsilon}$ & contact angle with consideration of assembly error \\
\hline$\delta_{i j k}$ & $\begin{array}{l}\text { the contact deformation of the } k^{\text {th }} \text { ball in the } j^{\text {th }} \text { groove of the } i^{\text {th }} \\
\text { carriage }\end{array}$ \\
\hline$Q_{i j k}$ & the contact force of the $k^{\text {th }}$ ball in the $j^{\text {th }}$ groove of the $i^{\text {th }}$ carriage \\
\hline$\alpha_{i j k}$ & the contact angle of the $k^{\text {th }}$ ball in the $j^{\text {th }}$ groove of the $i^{\text {th }}$ carriage \\
\hline$\Delta x_{i j k}, \Delta y_{i j k}$ & $\begin{array}{l}\text { the contact deformation of the } k^{\text {th }} \text { ball along } x \text { and } y \text { directions } \\
\text { under external load }\end{array}$ \\
\hline$x, y$ & $\begin{array}{l}\text { the displacement of the platform along } x \text { and } y \text { axis under external } \\
\text { load }\end{array}$ \\
\hline$L_{x}, L_{y}$ & the distance between grooves along $x$ and $y$ directions \\
\hline$l_{x}, l_{y}$ & the distance between carriages along $x$ and $y$ directions \\
\hline & \\
\hline$F_{x}, F_{y}$ & total restoring force of platform along $x$, and $y$ axis \\
\hline$M_{x}, M_{y}, M_{z}$ & total restoring moment of platform about $x, y$ and $z$ \\
\hline$I_{x}, I_{y}, I_{z}$ & \\
\hline
\end{tabular}




\begin{tabular}{|ll|}
\hline $\mathrm{w}_{x}, \mathrm{w}_{y}, \mathrm{w}_{z}$ & width, length, and depth of platform \\
Subscripts & \\
$i$ & $i^{\text {th }}$ rail \\
$j$ & $j^{\text {th }}$ carriage \\
$k$ & $k^{\text {th } \text { ball }}$ \\
$x$ & $x$ axis \\
$y$ & $y$ axis \\
$z$ & $z$ axis \\
$a$ & Type $a$ assembly error \\
$b$ & Type $b$ assembly error \\
$c$ & Type $c$ assembly error \\
$d$ & Type $d$ assembly error \\
$e$ & Type $e$ assembly error \\
\hline
\end{tabular}

\section{Dynamic model and governing equations of motion}

\subsection{Dynamic modeling of assembly error of linear guidway}

As shown in Fig. 1, a typical linear guideway slide platform consists of four carriages, a platform and two rails. $O$ represents the geometric center of the platform when there exist no assembly error, and $O^{\prime}$ is the new geometric center of the platform under the action of assembly error. The time varying external force $F_{x}$ and $F_{y}$ are applied to the geometric center of the platform along $x$ and $y$ axis. Due to the platform can move along $z$ axis, the external force along $z$ axis is not considered. Besides, there exist three external moments $\left(M_{x}, M_{y}, M_{z}\right)$ acting on the platform about $x, y$ and $z$ axis.

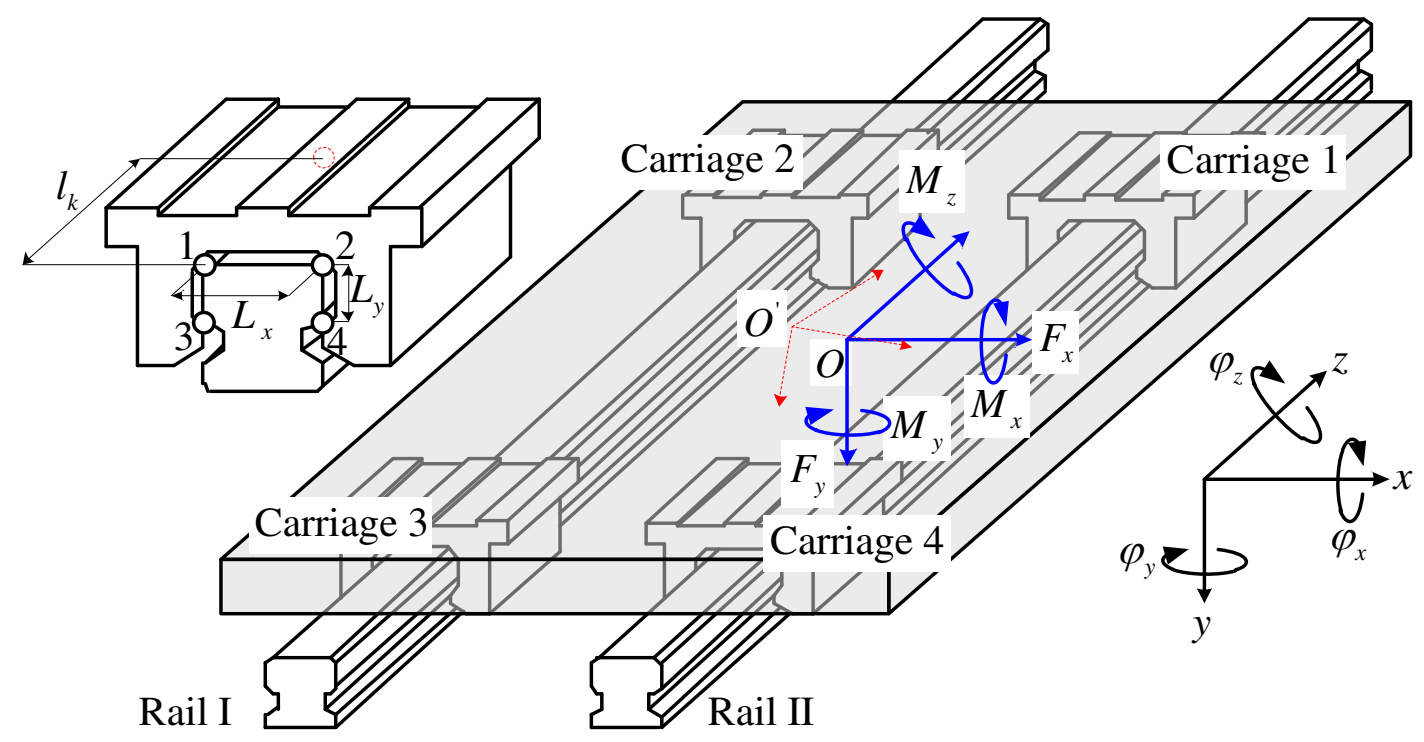

Fig.1 Schematic diagram of linear guideway slide platform.

As can be shown in Fig.2, Rail I is fixed by default, the assembly error of the platform is caused by the deviation of Rail II. The schematic of error-deformation relationship of balls for each type of assembly error can be shown in the Figure. The assembly errors are modeled as five types including: straightness error along $x$ and $y$ axis (Type $a$ and Type $b$ ), and rotation error about $x, y$ and $z$ axis (Type $c$, Type $d$ and Type $e$ ). It is noteworthy that the deviation of Rail II along $z$ axis is neglect in Fig. 2, this is because the dynamic and static simulations are carried out based on the state of stress equilibrium of the system. In other words, under the action of any types of assembly error and no external applied on the platform, the sum of the restoring force and the sum of the restoring moment of the system are equal to zero. The method of 
modeling five types of assembly error is achieved by calculating the distance between the curvature center of grooves.

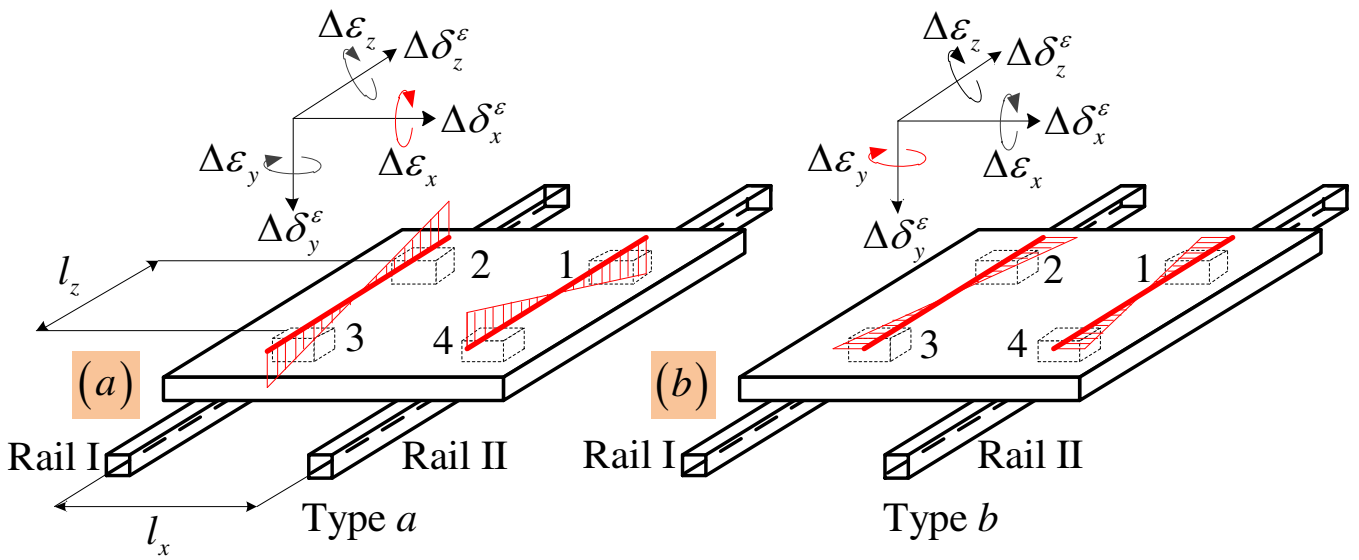

(c)
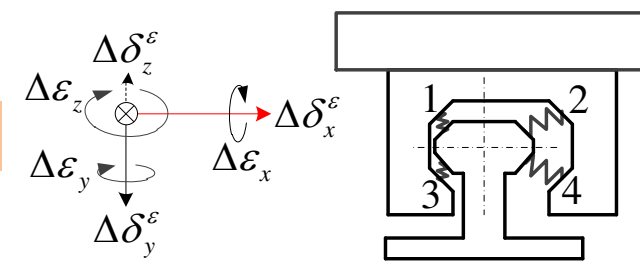

Type $c$
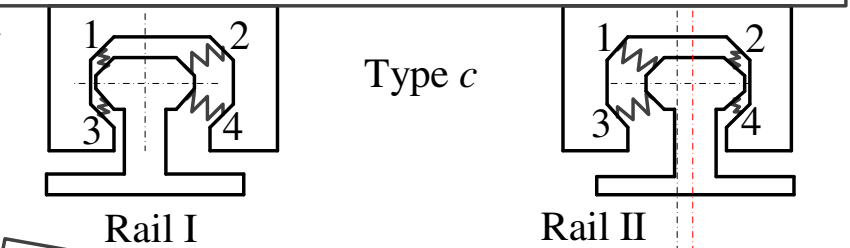

Rail II

(d)
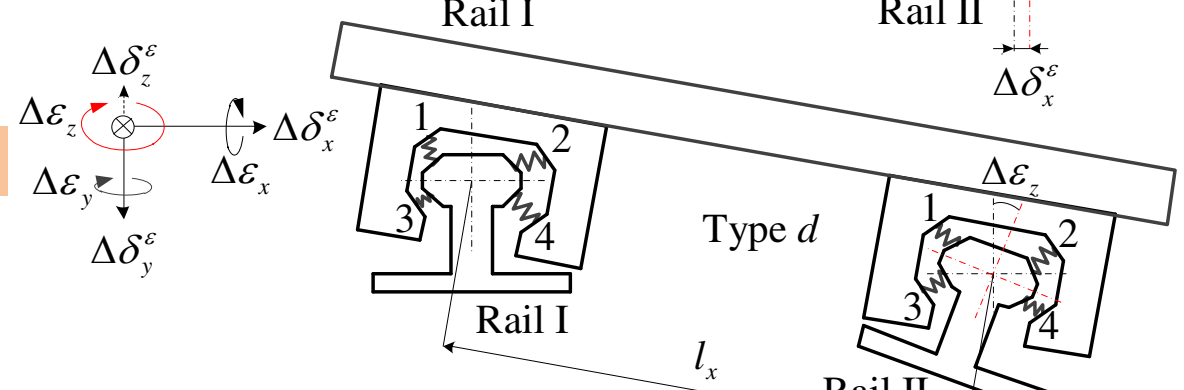

(e)

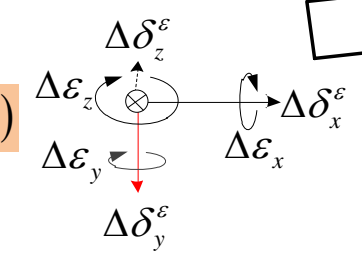

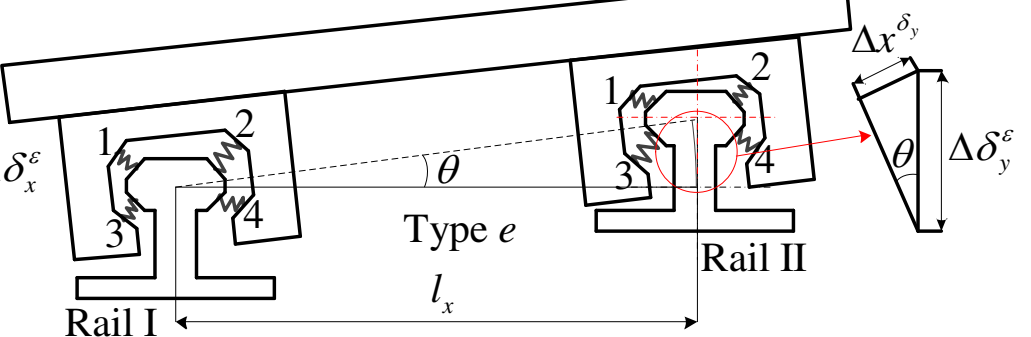

Fig.2 Schematic of different kinds of assembly errors of Rail II.(a) Type a, (b) Type b, (c) Type c, (d) Type d, (e) Type e

\subsubsection{Modeling of Type $a$ error}

For type $a$, the assembly error induced contact deformation along $x$ axis satisfies

$$
\Delta x_{i j k}^{a}=0
$$

and the contact deformation along $y$ axis of the $k^{\text {th }}$ ball in $j^{\text {th }}$ groove of $i^{\text {th }}$ carriage can be calculated as follows

$$
\Delta y_{i j k}^{a}=(-1)^{i}\left\{\begin{array}{cc}
\Delta \varepsilon_{x}\left(0.5 l_{z}+l_{k}\right) & j=1,2 \\
-\Delta \varepsilon_{x}\left(0.5 l_{z}+l_{k}\right) & j=3,4
\end{array}\right.
$$

where $\Delta \varepsilon_{x}$ represents the rotational error of rail II around $x$ axis. $l_{k}$ is the distance between the $k^{\text {th }}$ ball and the geometric center along the $x$ axis in a carriage, and the 
expression can be given by

$$
l_{k}=-0.5 N_{b} r_{b}+r_{b}(k-1)
$$

\subsubsection{Modeling of Type $b$ error}

For type $b$, the assembly error induced contact deformation along $y$ axis is equal to zero

$$
\Delta y_{i j k}^{b}=0
$$

and the assembly error induced contact deformation along $x$ axis of the $k^{\text {th }}$ ball in $j^{\text {th }}$ groove of $i^{\text {th }}$ carriage can be calculated by

$$
\Delta x_{i j k}^{a}=(-1)^{i+1}\left\{\begin{array}{cc}
\Delta \varepsilon_{y}\left(0.5 l_{z}+l_{k}\right) & j=1,3 \\
-\Delta \varepsilon_{y}\left(0.5 l_{z}+l_{k}\right) & j=2,4
\end{array}\right.
$$

where $\Delta \varepsilon_{y}$ represents the rotational assembly error of rail II around $y$ axis.

\subsubsection{Modeling of Type $c$ error}

For type $c$, the assembly error induced contact deformation along $y$ axis is zero

$$
\Delta y_{i j k}^{c}=0
$$

For carriage 1 and carriage 4, the contact deformation of the $k^{\text {th }}$ ball in $j^{\text {th }}$ groove caused by type $c$ assembly error along $x$ axis can be given by

$$
\Delta x_{i j k}^{c}=\left\{\begin{array}{cc}
\Delta \delta_{x}^{\varepsilon} & i=1,4 j=2,4 \\
-\Delta \delta_{x}^{\varepsilon} & i=1,4 j=1,3
\end{array}\right.
$$

and for carriage 2 and carriage 3 , the deformation is

$$
\Delta x_{i j k}^{c}=\left\{\begin{array}{cc}
-\Delta \delta_{x}^{\varepsilon} & i=2,3 j=2,4 \\
\Delta \delta_{x}^{\varepsilon} & i=2,3 j=1,3
\end{array}\right.
$$

where $\Delta \delta_{x}^{\varepsilon}$ represents the straightness error of rail II along $x$ axis.

\subsubsection{Modeling of Type $d$ error}

The schematic of type $d$ assembly error is shown in Fig. 2(d). According to the load-displacement relationship shown in Fig. 3, the contact deformation of balls caused by type $d$ assembly error can be obtained. For grooves 1 and grooves 4 in carriage 1 and carriage 4, the deformation along $x$ and $y$ axis can be obtained as follows:

$$
\left\{\begin{array}{l}
\Delta x_{i j k}^{d}=O_{r 1}^{\prime} O_{r 1} \Delta \varepsilon_{z} \cos \left(\frac{\left(\pi-\Delta \varepsilon_{z}\right)}{2}-\arctan \frac{L_{y}}{L_{x}}\right) \quad i=1,4 j=1,4 \\
\Delta y_{i j k}^{d}=O_{r 2}^{\prime} O_{r 2} \Delta \varepsilon_{z} \sin \left(\frac{\left(\pi-\Delta \varepsilon_{z}\right)}{2}-\arctan \frac{L_{y}}{L_{x}}\right) \quad i=1,4 j=1,4
\end{array}\right.
$$




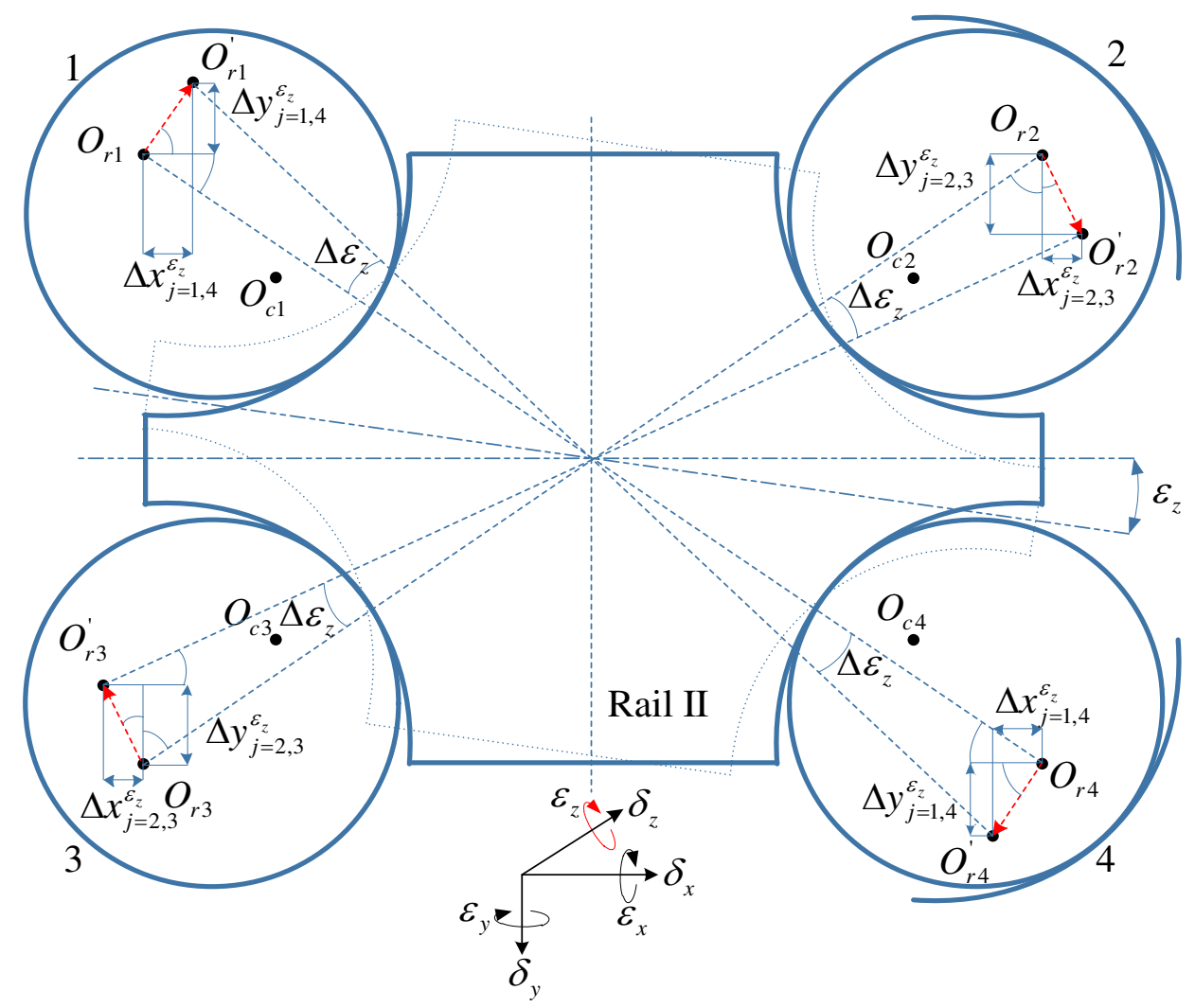

Fig. 3 load-deformation relationship of rail II for type $d$ assembly error

For groove 2 and groove 3 in carriage 1 and carriage 4, the deformation along $x$ and $y$ axis can be calculated by:

$$
\left\{\begin{array}{l}
\Delta x_{i j k}^{d}=O_{r 3}^{\prime} O_{r 3} \Delta \varepsilon_{z} \sin \left(\frac{\left(\pi-\Delta \varepsilon_{z}\right)}{2}-\arctan \frac{L_{x}}{L_{y}}\right) \quad i=1,4 j=2,3 \\
\Delta y_{i j k}^{d}=O_{r 4}^{\prime} O_{r 4} \Delta \varepsilon_{z} \cos \left(\frac{\left(\pi-\Delta \varepsilon_{z}\right)}{2}-\arctan \frac{L_{x}}{L_{y}}\right) \quad i=1,4 j=2,3
\end{array}\right.
$$

where $\Delta \varepsilon_{z}$ represents the rotation assembly error of rail II. Similarly, for groove 1 and groove 4 in carriage 2 and carriage 3 the contact deformation of balls along $x$ and $y$ axis can be expressed by

$$
\left\{\begin{array}{l}
\Delta x_{i j k}^{d}=O_{r 1}^{\prime} O_{r 1} \Delta \varepsilon_{z} \sin \left(\frac{\left(\pi-\Delta \varepsilon_{z}\right)}{2}-\arctan \frac{L_{x}}{L_{y}}\right) \quad i=2,3 j=1,4 \\
\Delta y_{i j k}^{d}=O_{r 2}^{\prime} O_{r 2} \Delta \varepsilon_{z} \cos \left(\frac{\left(\pi-\Delta \varepsilon_{z}\right)}{2}-\arctan \frac{L_{x}}{L_{y}}\right) \quad i=2,3 j=1,4
\end{array}\right.
$$

and for groove 2 and groove 3 in carriage 2 and 3 , the contact deformation of balls along $x$ and $y$ axis is

$$
\left\{\begin{array}{l}
\Delta x_{i j k}^{d}=O_{r 3}^{\prime} O_{r 3} \Delta \varepsilon_{z} \cos \left(\frac{\left(\pi-\Delta \varepsilon_{z}\right)}{2}-\arctan \frac{L_{y}}{L_{x}}\right) \quad i=2,3 j=2,3 \\
\Delta y_{i j k}^{d}=O_{r 4}^{\prime} O_{r 4} \Delta \varepsilon_{z} \sin \left(\frac{\left(\pi-\Delta \varepsilon_{z}\right)}{2}-\arctan \frac{L_{y}}{L_{x}}\right) \quad i=2,3 j=2,3
\end{array}\right.
$$




\subsubsection{Modeling of Type $e$ error}

As shown in Fig. 2(e), according to the geometric relationship, the incline angle $\theta$ can be calculated as follows:

$$
\theta=\arctan \frac{\Delta \delta_{y}^{\varepsilon}}{l_{x}}
$$

As can be seen in Fig. 2(e), the assembly error induced deformation along $y$ axis is

$$
\Delta y_{i j k}^{e}=0
$$

In carriage 1 and carriage 4, the contact deformation of the $k^{\text {th }}$ ball caused by type $e$ assembly error can be given by

$$
\Delta x_{i j k}^{e}=\left\{\begin{array}{ccc}
-\Delta \delta_{y}^{\varepsilon} \sin \theta & i=1,4 & j=1,3 \\
\Delta \delta_{y}^{\varepsilon} \sin \theta & i=1,4 & j=2,4
\end{array}\right.
$$

in carriage 2 and carriage 3 , the contact deformation of the $k^{\text {th }}$ ball caused by type $e$ assembly error can be given by

$$
\Delta x_{i j k}^{e}=\left\{\begin{array}{ccc}
\Delta \delta_{y}^{\varepsilon} \sin \theta & i=2,3 & j=1,3 \\
-\Delta \delta_{y}^{\varepsilon} \sin \theta & i=2,3 & j=2,4
\end{array}\right.
$$

where $\Delta \delta_{y}^{\varepsilon}$ represents the straightness error of rail II along $y$ axis.

In summary, the contact deformation along $x$ and $y$ axis caused by any given type of assembly error can be expressed by

$$
\left\{\begin{array}{l}
\Delta x_{i j k}^{\varepsilon}=\Delta x_{i j k}^{a}+\Delta x_{i j k}^{b}+\Delta x_{i j k}^{c}+\Delta x_{i j k}^{d}+\Delta x_{i j k}^{e} \\
\Delta y_{i j k}^{\varepsilon}=\Delta y_{i j k}^{a}+\Delta y_{i j k}^{b}+\Delta y_{i j k}^{c}+\Delta y_{i j k}^{d}+\Delta y_{i j k}^{e}
\end{array}\right.
$$

and the assembly error induced pre-deformation of the $k^{\text {th }}$ ball in $j^{\text {th }}$ groove of $i^{\text {th }}$ carriage can be given by

$$
\delta_{0}^{\varepsilon}=0.5\left(\sqrt{\Delta x_{i j k}^{\varepsilon 2}+\Delta y_{i j k}^{\varepsilon}}-S_{0}+2 \delta_{0}\right)
$$

where $\delta_{0}$ represents the contact deformation caused by preload. For the $k^{\text {th }}$ ball in $j^{\text {th }}$ groove of $i^{\text {th }}$ carriage, the initial distance between the groove curvature centers of carriage and rail can be expressed by

$$
S_{0}^{\varepsilon}=r_{c g}+r_{r g}-2 r_{b}+2 \delta_{0}^{\varepsilon}
$$

where $r \mathrm{~b}$ is the diameter of ball, $r_{\mathrm{cg}}$ and $r_{\mathrm{rg}}$ represent the groove curvature diameter of carriage and rail, and the expression of initial contact angle is

$$
\alpha_{0}^{\varepsilon}=\arctan \frac{\Delta y_{i j k}}{\Delta x_{i j k}}
$$

\subsection{Restoring force of linear guide slide platform}

In section 2.1, the expression for the pre-deformation of each ball was given, and the initial distance between groove curvature centers and the contact angle of each ball are derived. In this section, the expressions of assembly errors will be used to calculate the restoring force and restoring moment of the linear guide slide platform.

The contact deformation of the $k^{\text {th }}$ ball in each groove of Carriage 1 along $x$ and $y$ axis can be given by

$$
\left\{\begin{array}{l}
\Delta x_{\mathrm{I} 1 k}=x+\varphi_{y}\left(0.5 l_{z}+l_{k}\right)+S_{0}^{\varepsilon} \cos \alpha_{0}^{\varepsilon} \\
\Delta y_{\mathrm{I} 1 k}=y-\varphi_{x}\left(0.5 l_{z}+l_{k}\right)+0.5 \varphi_{z}\left(l_{x}-L_{x}\right)+S_{0}^{\varepsilon} \sin \alpha_{0}^{\varepsilon}
\end{array}\right.
$$




$$
\begin{aligned}
& \left\{\begin{array}{l}
\Delta x_{\mathrm{I} 2 k}=-x-\varphi_{y}\left(0.5 l_{z}+l_{k}\right)+S_{0}^{\varepsilon} \cos \alpha_{0}^{\varepsilon} \\
\Delta y_{\mathrm{I} 2 k}=y-\varphi_{x}\left(0.5 l_{z}+l_{k}\right)+0.5 \varphi_{z}\left(l_{x}+L_{x}\right)+S_{0}^{\varepsilon} \sin \alpha_{0}^{\varepsilon}
\end{array}\right. \\
& \left\{\begin{array}{l}
\Delta x_{\mathrm{I} 3 \mathrm{k}}=x+\varphi_{y}\left(0.5 l_{z}+l_{k}\right)+S_{0}^{\varepsilon} \cos \alpha_{0}^{\varepsilon} \\
\Delta y_{\mathrm{I} 3 \mathrm{k}}=-y+\varphi_{x}\left(0.5 l_{z}+l_{k}\right)-0.5 \varphi_{z}\left(l_{x}-L_{x}\right)+S_{0}^{\varepsilon} \sin \alpha_{0}^{\varepsilon}
\end{array}\right. \\
& \left\{\begin{array}{l}
\Delta x_{\mathrm{I} 4 \mathrm{k}}=-x-\varphi_{y}\left(0.5 l_{z}+l_{k}\right)+S_{0}^{\varepsilon} \cos \alpha_{0}^{\varepsilon} \\
\Delta y_{\mathrm{I} 4 \mathrm{k}}=-y+\varphi_{x}\left(0.5 l_{z}+l_{k}\right)-0.5 \varphi_{z}\left(l_{x}+L_{x}\right)+S_{0}^{\varepsilon} \sin \alpha_{0}^{\varepsilon}
\end{array}\right.
\end{aligned}
$$

For Carriage 2 is

$$
\begin{aligned}
& \left\{\begin{array}{l}
\Delta x_{\mathrm{II} 1 \mathrm{k}}=x+\varphi_{y}\left(0.5 l_{z}+l_{k}\right)+S_{0}^{\varepsilon} \cos \alpha_{0}^{\varepsilon} \\
\Delta y_{\mathrm{III \textrm {k }}}=y-\varphi_{x}\left(0.5 l_{z}+l_{k}\right)-0.5 \varphi_{z}\left(l_{x}+L_{x}\right)+S_{0}^{\varepsilon} \sin \alpha_{0}^{\varepsilon}
\end{array}\right. \\
& \left\{\begin{array}{l}
\Delta x_{\mathrm{II} 2 \mathrm{k}}=-x-\varphi_{y}\left(0.5 l_{z}+l_{k}\right)+S_{0}^{\varepsilon} \cos \alpha_{0}^{\varepsilon} \\
\Delta y_{\mathrm{II} 2 \mathrm{k}}=y-\varphi_{x}\left(0.5 l_{z}+l_{k}\right)-0.5 \varphi_{z}\left(l_{x}-L_{x}\right)+S_{0}^{\varepsilon} \sin \alpha_{0}^{\varepsilon}
\end{array}\right. \\
& \left\{\begin{array}{l}
\Delta x_{\mathrm{II} 3 \mathrm{k}}=x+\varphi_{y}\left(0.5 l_{z}+l_{k}\right)+S_{0}^{\varepsilon} \cos \alpha_{0}^{\varepsilon} \\
\Delta y_{\mathrm{II} \mathrm{k}}=-y+\varphi_{x}\left(0.5 l_{z}+l_{k}\right)+0.5 \varphi_{z}\left(l_{x}+L_{x}\right)+S_{0}^{\varepsilon} \sin \alpha_{0}^{\varepsilon}
\end{array}\right. \\
& \left\{\begin{array}{l}
\Delta x_{\mathrm{II} 4 \mathrm{k}}=-x-\varphi_{y}\left(0.5 l_{z}+l_{k}\right)+S_{0}^{\varepsilon} \cos \alpha_{0}^{\varepsilon} \\
\Delta y_{\mathrm{II} 4 \mathrm{k}}=-y+\varphi_{x}\left(0.5 l_{z}+l_{k}\right)+0.5 \varphi_{z}\left(l_{x}-L_{x}\right)+S_{0}^{\varepsilon} \sin \alpha_{0}^{\varepsilon}
\end{array}\right.
\end{aligned}
$$

For Carriage 3, the contact deformation of the $k^{\text {th }}$ ball along $x$ and $y$ axis can be calculated, respectively

$$
\begin{aligned}
& \left\{\begin{array}{l}
\Delta x_{\mathrm{III} 1 \mathrm{k}}=x-\varphi_{y}\left(0.5 l_{z}+l_{k}\right)+S_{0}^{\varepsilon} \cos \alpha_{0}^{\varepsilon} \\
\Delta y_{\mathrm{III} 1 \mathrm{k}}=y+\varphi_{x}\left(0.5 l_{z}+l_{k}\right)-0.5 \varphi_{z}\left(l_{x}+L_{x}\right)+S_{0}^{\varepsilon} \sin \alpha_{0}^{\varepsilon}
\end{array}\right. \\
& \left\{\begin{array}{l}
\Delta x_{\mathrm{III} 2 \mathrm{k}}=-x+\varphi_{y}\left(0.5 l_{z}+l_{k}\right)+S_{0}^{\varepsilon} \cos \alpha_{0}^{\varepsilon} \\
\Delta y_{\mathrm{III} 2 \mathrm{k}}=y+\varphi_{x}\left(0.5 l_{z}+l_{k}\right)-0.5 \varphi_{z}\left(l_{x}-L_{x}\right)+S_{0}^{\varepsilon} \sin \alpha_{0}^{\varepsilon}
\end{array}\right. \\
& \left\{\begin{array}{l}
\Delta x_{\mathrm{III} 3 \mathrm{k}}=x-\varphi_{y}\left(0.5 l_{z}+l_{k}\right)+S_{0}^{\varepsilon} \cos \alpha_{0}^{\varepsilon} \\
\Delta y_{\mathrm{III} 3 \mathrm{k}}=-y-\varphi_{x}\left(0.5 l_{z}+l_{k}\right)+0.5 \varphi_{z}\left(l_{x}+L_{x}\right)+S_{0}^{\varepsilon} \sin \alpha_{0}^{\varepsilon}
\end{array}\right. \\
& \left\{\begin{array}{l}
\Delta x_{\mathrm{III} 4 \mathrm{k}}=-x+\varphi_{y}\left(0.5 l_{z}+l_{k}\right)+S_{0}^{\varepsilon} \cos \alpha_{0}^{\varepsilon} \\
\Delta y_{\mathrm{III} 4 \mathrm{k}}=-y-\varphi_{x}\left(0.5 l_{z}+l_{k}\right)+0.5 \varphi_{z}\left(l_{x}-L_{x}\right)+S_{0}^{\varepsilon} \sin \alpha_{0}^{\varepsilon}
\end{array}\right.
\end{aligned}
$$

And for Carriage 4 the expression of the $k^{\text {th }}$ ball along $x$ and $y$ axis are as follows

$$
\begin{aligned}
& \left\{\begin{array}{l}
\Delta x_{\mathrm{IV} 1 \mathrm{k}}=x-\varphi_{y}\left(0.5 l_{z}+l_{k}\right)+S_{0}^{\varepsilon} \cos \alpha_{0}^{\varepsilon} \\
\Delta y_{\mathrm{IV} 1 \mathrm{k}}=y+\varphi_{x}\left(0.5 l_{z}+l_{k}\right)+0.5 \varphi_{z}\left(l_{x}-L_{x}\right)+S_{0}^{\varepsilon} \sin \alpha_{0}^{\varepsilon}
\end{array}\right. \\
& \left\{\begin{array}{l}
\Delta x_{\mathrm{IV} 2 \mathrm{k}}=-x+\varphi_{y}\left(0.5 l_{z}+l_{k}\right)+S_{0}^{\varepsilon} \cos \alpha_{0}^{\varepsilon} \\
\Delta y_{\mathrm{IV} 2 \mathrm{k}}=y+\varphi_{x}\left(0.5 l_{z}+l_{k}\right)+0.5 \varphi_{z}\left(l_{x}+L_{x}\right)+S_{0}^{\varepsilon} \sin \alpha_{0}^{\varepsilon}
\end{array}\right. \\
& \left\{\begin{array}{l}
\Delta x_{\mathrm{IV} 3 \mathrm{k}}=x-\varphi_{y}\left(0.5 l_{z}+l_{k}\right)+S_{0}^{\varepsilon} \cos \alpha_{0}^{\varepsilon} \\
\Delta y_{\mathrm{IV} 3 \mathrm{k}}=-y-\varphi_{x}\left(0.5 l_{z}+l_{k}\right)-0.5 \varphi_{z}\left(l_{x}-L_{x}\right)+S_{0}^{\varepsilon} \sin \alpha_{0}^{\varepsilon}
\end{array}\right.
\end{aligned}
$$




$$
\left\{\begin{array}{l}
\Delta x_{\mathrm{IV} 4 \mathrm{k}}=-x+\varphi_{y}\left(0.5 l_{z}+l_{k}\right)+S_{0}^{\varepsilon} \cos \alpha_{0}^{\varepsilon} \\
\Delta y_{\mathrm{IV} 4 \mathrm{k}}=-y-\varphi_{x}\left(0.5 l_{z}+l_{k}\right)-0.5 \varphi_{z}\left(l_{x}+L_{x}\right)+S_{0}^{\varepsilon} \sin \alpha_{0}^{\varepsilon}
\end{array}\right.
$$

where $x, y$ represent the displacement of the geometric center along $x$ and $y$ axis, and $\varphi_{x}, \varphi_{y}$ and $\varphi_{z}$ represent the angular displacement about $x, y$ and $z$ axis. $L_{x}$ is the distance between grooves in a carriage along $x$ axis, which can be shown in Fig. 1. Then, the contact deformation of the $k^{\text {th }}$ ball in $j^{\text {th }}$ groove of $i^{\text {th }}$ carriage under external load can be expressed as

$$
\delta_{i j k}=0.5\left(\sqrt{\Delta x_{i j k}{ }^{2}+\Delta y_{i j k}{ }^{2}}-S_{0}^{\varepsilon}+2 \delta_{0}^{\varepsilon}\right)
$$

According to the Hertz contact theory [28], the contact force of rolling ball can be given by

$$
Q_{i j k}=K \delta_{i j k}^{3 / 2}
$$

where $\mathrm{K}$ is the Hertz contact stiffness, and the contact angle is

$$
\alpha_{i j k}=\arcsin \frac{\Delta y_{i j k}}{\Delta x_{i j k}}
$$

The total restoring force along $x$ axis of the vibration system can be given by

$$
F_{x}=\sum_{i}^{4} \sum_{i}^{4} \sum_{k}^{N_{b}}\left\{\begin{array}{cc}
Q_{i j k} \cos \alpha & j=1,3 \\
-Q_{i j k} \cos \alpha & j=2,4
\end{array}\right.
$$

The total restoring force along $y$ axis of the vibration system can be given by

$$
F_{y}=\sum_{i}^{4} \sum_{i}^{4} \sum_{k}^{N_{b}}\left\{\begin{array}{cc}
Q_{i j k} \sin \alpha & j=1,2 \\
-Q_{i j k} \sin \alpha & j=3,4
\end{array}\right.
$$

The total restoring moment about $x$ axis of the vibration system can be given by

$$
M_{x}=\sum_{i=1}^{4} \sum_{j=1}^{4} \sum_{k=1}^{N_{b}} \begin{cases}-\sum_{j=1,2} Q_{i j k} \sin \alpha\left(l_{z}+l_{k}\right) & \\ +\sum_{j=3,4} Q_{i j k} \sin \alpha\left(l_{z}+l_{k}\right) & i=1,2 \\ \sum_{j=1,2} Q_{i j k} \sin \alpha\left(l_{z}-l_{k}\right) & \\ -\sum_{j=3,4} Q_{i j k} \sin \alpha\left(l_{z}-l_{k}\right) & i=3,4\end{cases}
$$

The total restoring moment about $y$ axis of the vibration system can be given by

$$
M_{y}=\sum_{i=1}^{4} \sum_{j=1}^{4} \sum_{k=1}^{N_{b}} \begin{cases}\sum_{j=1,2} Q_{i j k} \cos \alpha\left(l_{z}+l_{k}\right) & \\ -\sum_{j=3,4} Q_{i j k} \cos \alpha\left(l_{z}+l_{k}\right) & i=1,2 \\ -\sum_{j=1,2} Q_{i j k} \cos \alpha\left(l_{z}-l_{k}\right) & \\ +\sum_{j=3,4} Q_{i j k} \cos \alpha\left(l_{z}-l_{k}\right) & i=3,4\end{cases}
$$

The total restoring moment about $z$ axis of the vibration system can be given by 


$$
M_{z}=\sum_{i=1}^{4} \sum_{j=1}^{4} \sum_{k=1}^{N_{b}}\left\{\begin{array}{l}
\sum_{j=1,2} Q_{i j k} \cos \alpha\left(l_{z}+(-1)^{j} l_{k}\right) \\
-\sum_{j=3,4} Q_{i j k} \cos \alpha\left(l_{z}+(-1)^{j} l_{k}\right) \\
-\sum_{j=1,2} Q_{i j k} \cos \alpha\left(l_{z}-(-1)^{j} l_{k}\right) \\
+\sum_{j=3,4} Q_{i j k} \cos \alpha\left(l_{z}-(-1)^{j} l_{k}\right)
\end{array} \quad i=2,3\right.
$$

In this study, for any given type and value of assembly error and no external load applied on the system, the restoring force and restoring moment $F_{x}, F_{y}, M_{x}, M_{y}$, and $M_{z}$ satisfies

$$
\begin{aligned}
& F_{x}\left(\begin{array}{l}
x=0, y=0, \varphi_{x}=0, \varphi_{y}=0, \varphi_{z}=0, \\
\Delta \delta_{x}^{\varepsilon}, \Delta \delta_{x}^{\varepsilon}, \Delta \varepsilon_{x}^{\varepsilon}, \Delta \varepsilon_{x}^{\varepsilon}, \Delta \varepsilon_{x}^{\varepsilon}
\end{array}\right)=0 \\
& F_{y}\left(\begin{array}{l}
x=0, y=0, \varphi_{x}=0, \varphi_{y}=0, \varphi_{z}=0, \\
\Delta \delta_{x}^{\varepsilon}, \Delta \delta_{x}^{\varepsilon}, \Delta \varepsilon_{x}^{\varepsilon}, \Delta \varepsilon_{x}^{\varepsilon}, \Delta \varepsilon_{x}^{\varepsilon}
\end{array}\right)=0 \\
& M_{x}\left(\begin{array}{l}
x=0, y=0, \varphi_{x}=0, \varphi_{y}=0, \varphi_{z}=0, \\
\Delta \delta_{x}^{\varepsilon}, \Delta \delta_{x}^{\varepsilon}, \Delta \varepsilon_{x}^{\varepsilon}, \Delta \varepsilon_{x}^{\varepsilon}, \Delta \varepsilon_{x}^{\varepsilon}
\end{array}\right)=0 \\
& M_{y}\left(\begin{array}{l}
x=0, y=0, \varphi_{x}=0, \varphi_{y}=0, \varphi_{z}=0, \\
\Delta \delta_{x}^{\varepsilon}, \Delta \delta_{x}^{\varepsilon}, \Delta \varepsilon_{x}^{\varepsilon}, \Delta \varepsilon_{x}^{\varepsilon}, \Delta \varepsilon_{x}^{\varepsilon}
\end{array}\right)=0 \\
& M_{z}\left(\begin{array}{l}
x=0, y=0, \varphi_{x}=0, \varphi_{y}=0, \varphi_{z}=0, \\
\Delta \delta_{x}^{\varepsilon}, \Delta \delta_{x}^{\varepsilon}, \Delta \varepsilon_{x}^{\varepsilon}, \Delta \varepsilon_{x}^{\varepsilon}, \Delta \varepsilon_{x}^{\varepsilon}
\end{array}\right)=0
\end{aligned}
$$

According to [29], the moment of inertia of the platform about $x, y$ and $z$ axis can be calculated by

$$
\begin{aligned}
& I_{x}=\frac{1}{12} m\left(w^{2}+d^{2}\right) \\
& I_{y}=\frac{1}{12} m\left(w^{2}+h^{2}\right) \\
& I_{z}=\frac{1}{12} m\left(h^{2}+d^{2}\right)
\end{aligned}
$$

where $m$ is the mass of the platform, $h, w$, and $d$ represent the height, width, and length, respectively.

\subsection{Equations of motions}

In this section, considering the influence of different types of assembly error and external load, an equivalent nonlinear dynamic model of the linear guide slide platform can be simplified as a 5-degrees-of-freedom mass-spring-damper model. According to the schematic which is shown in Fig. 2, the governing equations of motion can be expressed as follows

$$
\begin{gathered}
m \ddot{x}+c_{x} \dot{x}+F_{x}=F_{x}(t) \\
m \ddot{y}+c_{y} \dot{y}+F_{y}=F_{y}(t) \\
I_{x} \ddot{\varphi}_{x}+c_{y} l_{x} \dot{\varphi}_{x}+M_{x}=M_{x}(t) \\
I_{y} \ddot{\varphi}_{y}+c_{x} l_{y} \dot{\varphi}_{y}+M_{y}=M_{y}(t)
\end{gathered}
$$




$$
I_{z} \ddot{\varphi}_{z}+c_{y} l_{z} \dot{\varphi}_{z}+M_{z}=M_{z}(t)
$$

where $c_{x}$ and $c_{y}$ represent the damping coefficient, and the value can be determined by the test in section 3.2, $x$ and $y$ represent the displacement of the geometric center of platform along $x$ and $y$ axis, $\varphi_{x}, \varphi_{y}$, and $\varphi_{z}$ represent the angular displacement of the geometric center of the platform about $x, y$, and $z$ axis. $F_{x}(t)$ and $F_{y}(t)$ represent the time varying exciting force, and satisfy $F_{x}(t)=F_{y}(t)=F_{0} \sin \omega t, \omega$ represents the excitation frequency. $M_{x}(t), M_{y}(t)$, and $M_{z}(t)$ are the external moment load about $x, y$ and $z$ axis, and satisfy $M_{x}(t)=M_{y}(t)=M_{y}(t)=l F_{0} \sin \omega \mathrm{t}$.

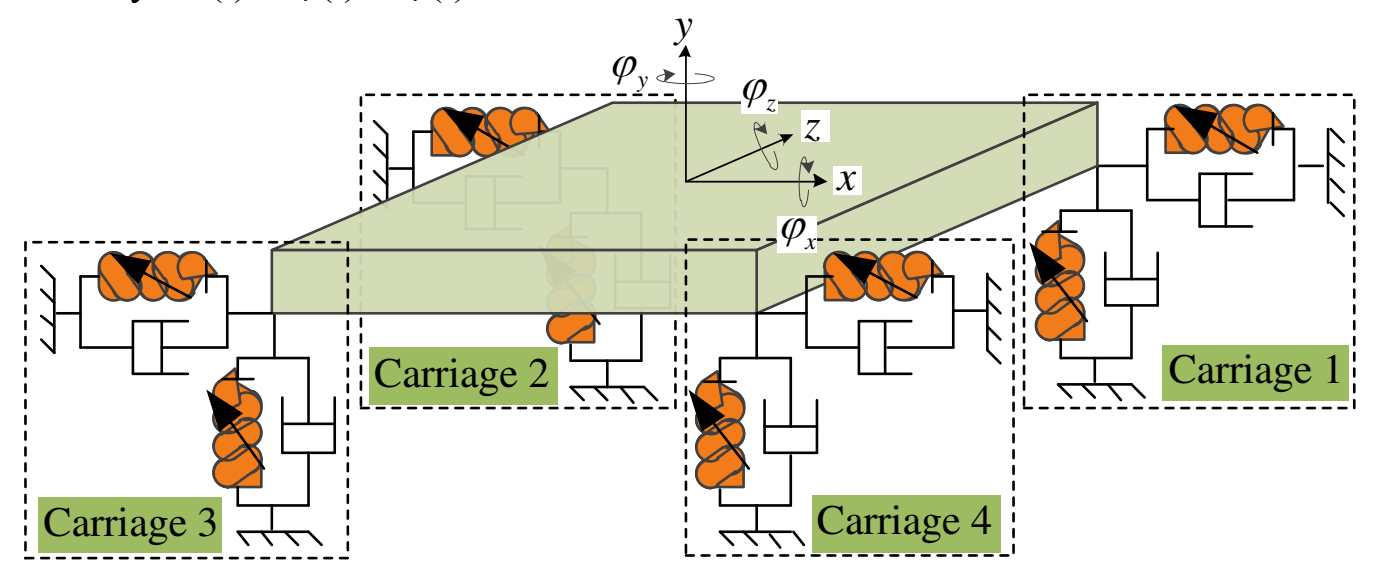

Fig. 2. Model schematic of linear guide slide platform

\section{Simulation and discussion}

In this section, the fourth order Runge Kutta method with fixed time step is used to analyze the governing equations of motion. The studied linear guide slide platform is defined in Table 1. To accelerate the calculation progress, the initial condition for differential equation is set to $\left[x, \dot{x}, y, \dot{y}, \varphi_{x}, \dot{\varphi}_{x}, \varphi_{y}, \dot{\varphi}_{y}, \varphi_{z}, \dot{\varphi}_{z}\right]=[0,0,0,0,0,0,0,0,0,0]$, and the value is determined by the mean of the solution when the steady state solution is obtained. In this study, steady state solution means the properties of the solution does not change over time. To obtain high precision solution, the absolute tolerance and relative tolerance are set to $10^{-4}$ and $10^{-4}$. To accelerate convergence, the time step in numerical simulation is set as $\Delta t=10^{-6}[30]$. The detailed procedure of the calculation is listed in Table. 2. To meet the needs of engineering reality, the max deflection distance of geometric center of each carriage caused by assembly error is less than $5 \mu \mathrm{m}$. In the simulation, the default system parameters are as follows: $F_{p}=623 \mathrm{~N}[31], F_{0}=100000 \mathrm{~N}$, and $\omega=4350 \mathrm{rad} / \mathrm{s}$.

Table. 1

Specifications of the linear guide.

\begin{tabular}{ll} 
Number of raceways of each carriage & 4 \\
Initial contact angle & $45^{\circ}$ \\
Number of loaded balls in a groove & 12 \\
Radius of ball $r b$ & $6.35 \mathrm{~mm}$ \\
Radius of groove $r$ & $3.2 \mathrm{~mm}$ \\
Elasticity modulus $E$ & $206 \mathrm{Gpa}$ \\
Preload & $623 \mathrm{~N}$ \\
Thickness of worktable & $30 \mathrm{~mm}$ \\
Distance between rails in $x$ direction $l_{x}$ & $350 \mathrm{~mm}$ \\
Distance between carriages in $z$ direction $l_{z}$ & $300 \mathrm{~mm}$ \\
Distance between grooves in $x$ direction in carriage $L_{x}$ & $32 \mathrm{~mm}$ \\
Distance between grooves in $y$ direction in carriage $L_{y}$ & $10 \mathrm{~mm}$ \\
\hline
\end{tabular}


Table. 2

Detailed procedure of the numerical calculation

1: given the initial parameters of the system

2: calculate the initial contact deformation of each ball according to the proposed method

3: ensure that the sum of the restoring force and restoring moment is equal to zero when there is no external load applied on the platform.

4: repeat

5: calculate the dynamic response under harmonic excitation

6: until stopping criterion is met

7: return solution

\subsection{Static analysis of linear guide slide platform}

Assembly error can influence the load distribution of linear guide, according to Archard wear theory [32], the ball with greater contact force is more likely to cause groove wear. In addition, the assembly error can affect the positioning accuracy of the platform. Therefore, the investigation of the influence of assembly error on the static characteristics is crucial.

Due to the force applied on each carriage is symmetric, taking carriage 1 as an example, Fig.3 shows the load distribution of balls in carriage 1. As shown in Fig. 3(a), with the increasing straightness assembly error $\Delta \delta_{x}^{\varepsilon}$, under the combined effect of preload and assembly error, the load of balls in groove 2 and groove 4 decrease to zero first, increase the assembly error further, the value of each ball remains unchanged due to the contact deformation is equal to zero. Furthermore, the balls in a groove are subjected to the same load.

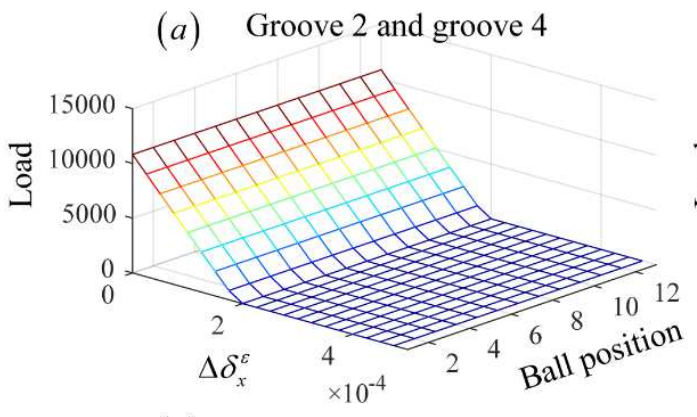

(c) Groove 1 and groove 2

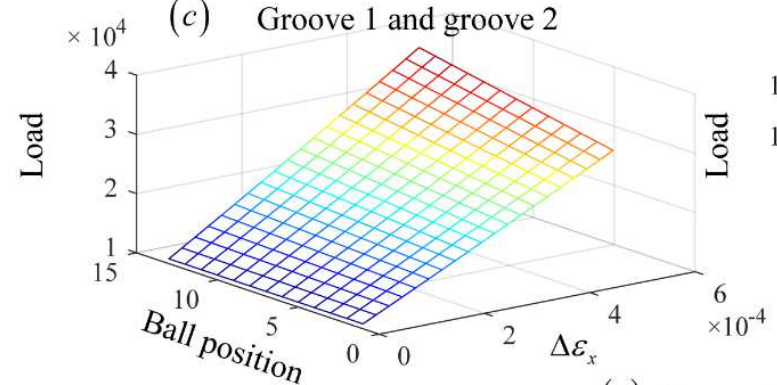

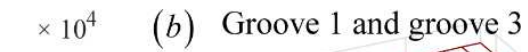

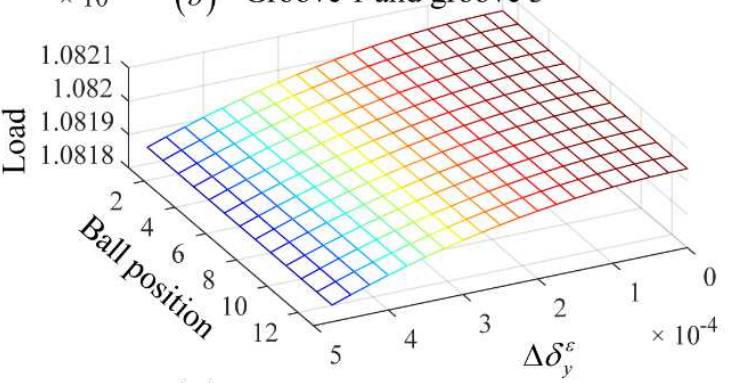

(d) Groove 1 and groove 3

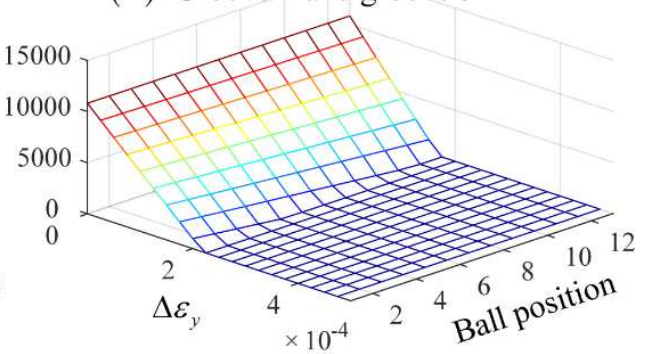

(e) Groove 1 and groove 4

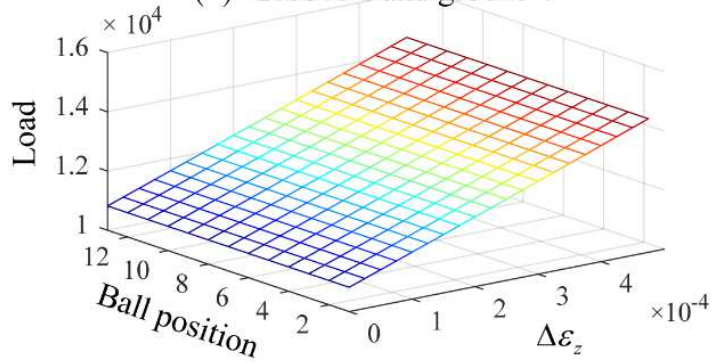

Fig. 3. Influence of different types assembly errors on load distribution of linear guide 
slide platform. (a) straightness error $\Delta \delta_{x}^{\varepsilon}$, (b) straightness error $\Delta \delta_{y}^{\varepsilon}$ (c) rotation error $\Delta \varepsilon_{x}$, (d) rotation error $\Delta \varepsilon_{y}$, (e) rotation error $\Delta \varepsilon_{z}$.

As can be seen in Fig. 3(b), for straightness assembly error $\Delta \delta_{y}^{\varepsilon}$, the contact deformation of balls in groove 1 and groove 3 decrease with $\Delta \delta_{y}^{\varepsilon}$, but the degree of linearity of the curve is less than Fig. 3(a). As shown in Fig. 3(c) and Fig. 3(d), for rotation assembly error $\Delta \varepsilon_{x}$, and $\Delta \varepsilon_{y}$, the balls in different positions in a groove are subjected to different loads, the further distance between the ball and the geometric center of carriage, the larger load it is subjected to. As shown in Fig. 3(e), for rotation assembly error $\Delta \varepsilon_{z}$, the loads of the balls in groove 1 and groove 4 increase with $\Delta \varepsilon_{z}$ more linearly.
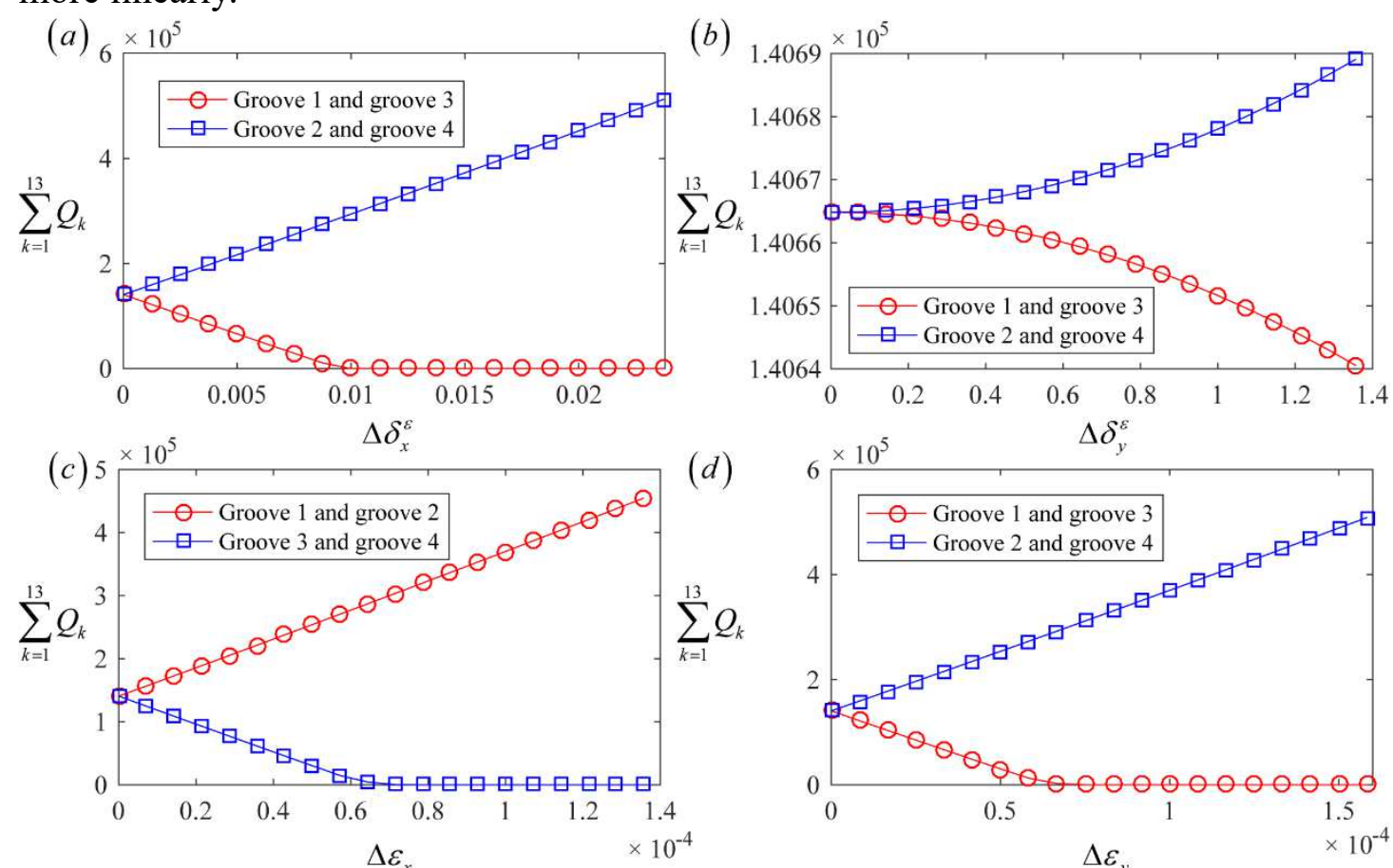

(d)
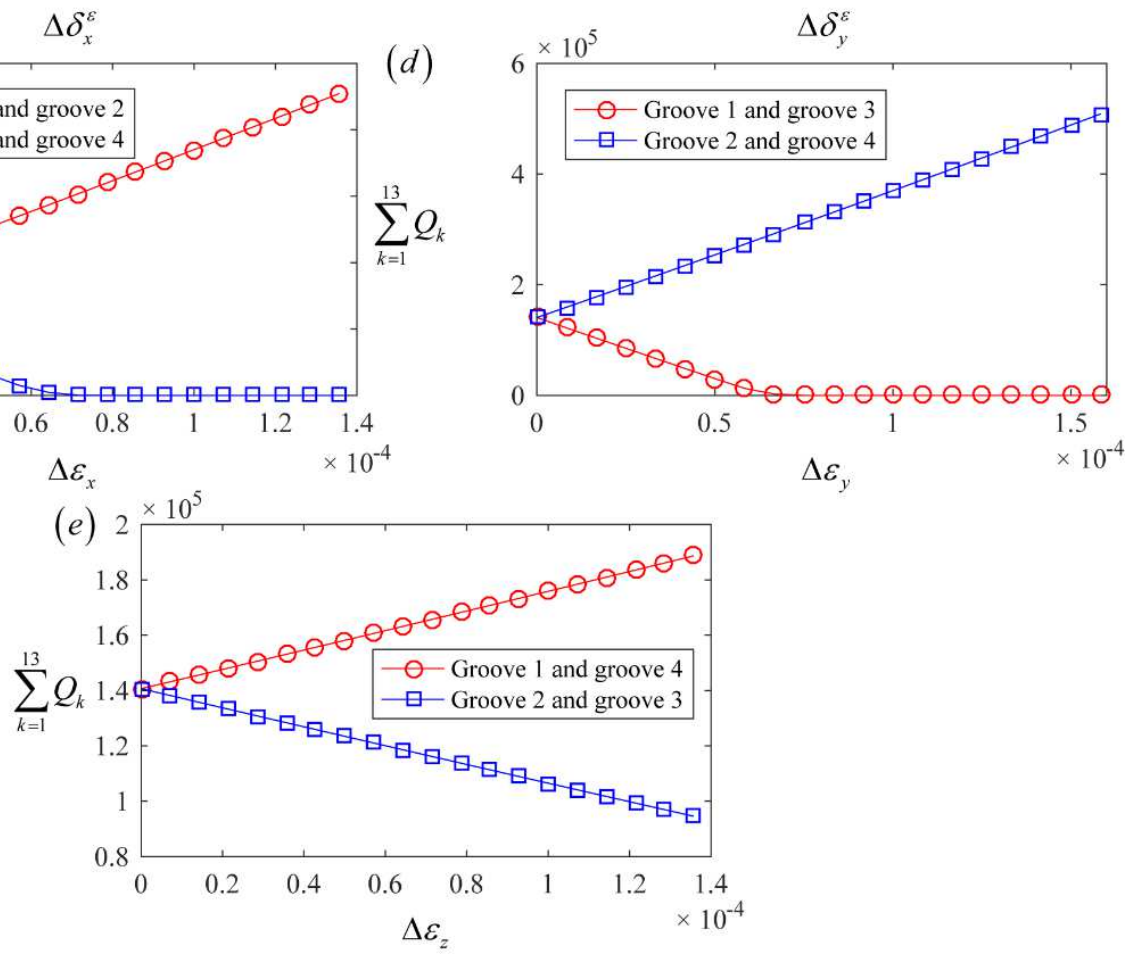

Fig. 4. Comparisons of load between grooves influence by (a) straightness assembly error $\Delta \delta_{x}^{\varepsilon}$, (b) straightness assembly error $\Delta \delta_{y}^{\varepsilon}$ (c) rotation error assembly $\Delta \varepsilon_{x}$, (d) rotation error assembly $\Delta \varepsilon_{y}$, (e) rotation error assembly $\Delta \varepsilon_{z}$.

The load distribution between balls in a specific groove influenced by assembly error has been discussed above. To further investigate the effect of assembly error on load distribution in a carriage, the comparison of $\Sigma Q_{k}$ between different grooves can be shown in Fig. 4. As shown in Fig. 4(a), the value of $\Sigma Q_{k}$ increase with $\Delta \delta_{x}^{\varepsilon}$ in groove 2 and groove 4 , and the value of $\Sigma Q_{k}$ in groove 1 and groove 3 decrease to zero and do not change with assembly error. This is because the value of preload decrease to zero and the ball is not deformed. In addition, the difference between the loads of left 
grooves (groove 1 and groove 3 ) and right grooves (groove 2 and groove 4) increase with assembly error $\Delta \delta_{x}^{\varepsilon}$, this means the balls in groove 1 and groove 3 are prone to cause wear under the action of assembly error $\Delta \delta_{x}^{\varepsilon}$. Similarly, left grooves (groove 1 and groove 3) shown in Fig. 4(b), upper grooves (groove 1 and groove 3) shown in Fig. 4(c), right grooves shown in Fig. 4(d), groove 1 and groove 4 shown in Fig. 4(e), these cases are prone to cause groove uneven wear.

The assembly error forms one of the biggest source of inaccuracy in machine tool [4]. Fig. 4 shows the relationship between the external static load and the positioning accuracy of the linear guide platform under the action of different assembly errors. It can be shown in the figure, due to the existence of initial error, the system with assembly error have lower positioning accuracy.
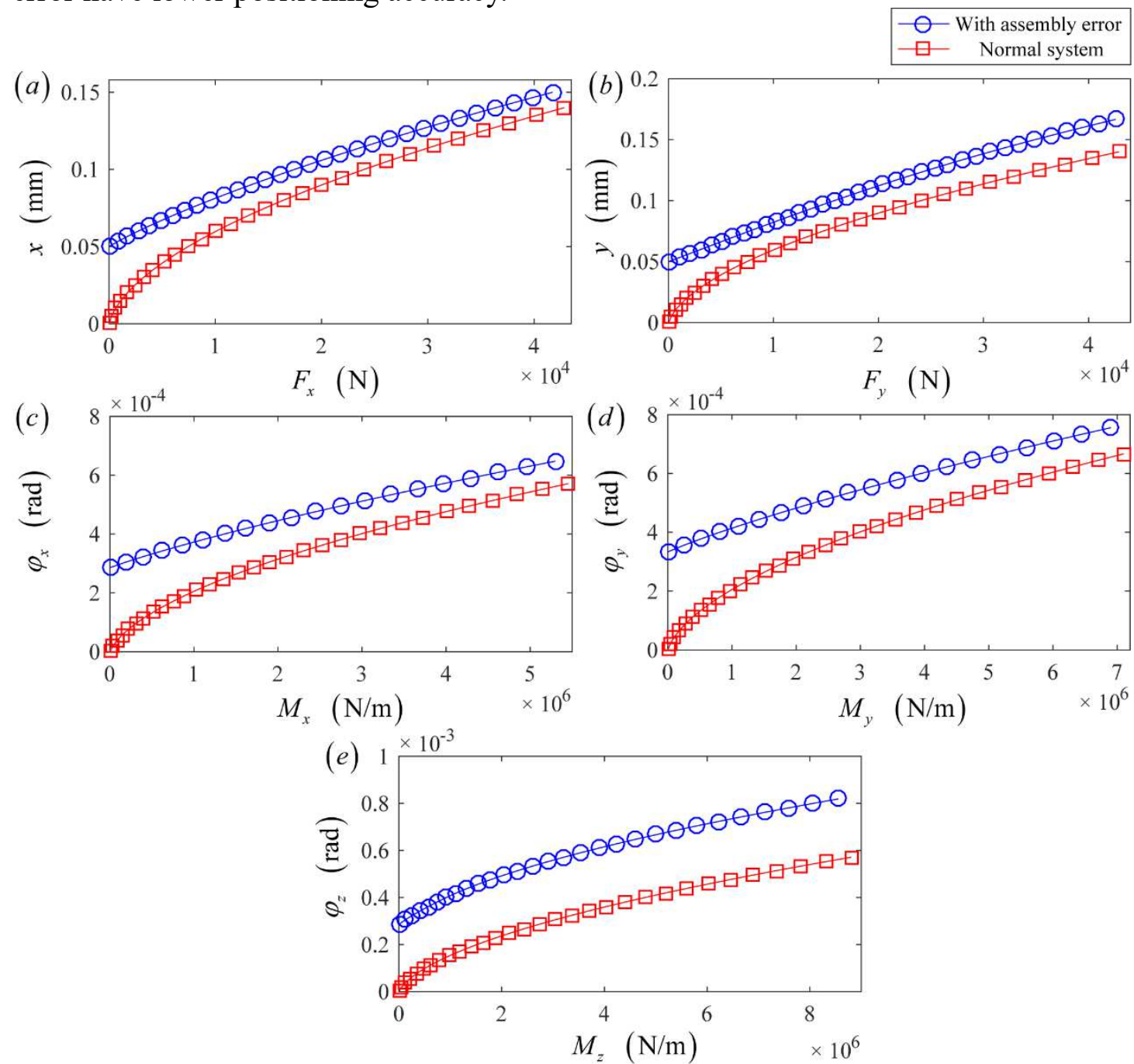

Fig. 4. Influence of different assembly errors on positioning accuracy. (a) straightness assembly error $\Delta \delta_{x}^{\varepsilon}$, (b) straightness assembly error $\Delta \delta_{y}^{\varepsilon}$ (c) rotation error assembly $\Delta \varepsilon_{x}$, (d) rotation error assembly $\Delta \varepsilon_{y}$, (e) rotation error assembly $\Delta \varepsilon_{z}$.

\subsection{Nonlinear dynamic analysis of linear guide slide platform}

In this section, to investigate the influence of different types of assembly errors on dynamic responses of linear guide slide platform, the bifurcation diagrams, the largest Lyapunov exponent (LLE), and 3-D frequency spectrums are presented with excitation frequency, excitation amplitude, assembly error, and preload as control parameters.

\subsubsection{The influence of excitation frequency}

Fig. 5 presents the corresponding bifurcation diagram of the linear guide slide 
platform vibration system with respect to excitation frequency under different types of assembly errors $\Delta \delta_{x}^{\varepsilon}, \Delta \delta_{y}^{\varepsilon}, \Delta \varepsilon_{x}, \Delta \varepsilon_{y}$, and $\Delta \varepsilon_{z}$. As shown in Fig. 5 (a), (b), (e) and (f), as the increase of excitation frequency, the motion of the system is periodic- 1 motion before resonance frequency, the values of LLE are less than zero which can be shown in Fig. 6 (a), (b), (e) and (f). With the increase of excitation frequency, the system enters to quasi-periodic motion and chaotic motion, the values of LLE alternate among negative, zero, and positive, this indicates that the excitation frequency is close to the resonance frequency and resonance phenomenon occurs. By increasing excitation frequency further, the motion of the system return to periodic-1 motion, and the values of LLE are less than zero. By comparing the bifurcation diagram shown in Fig. 5(a), (b), (e) and (f), there exist small differences between them, including the length of the interval of quasi-periodic motion. For rotation assembly error $\Delta \delta_{x}^{\varepsilon}$ and $\Delta \delta_{y}^{\varepsilon}$ which can be shown in Fig. 5(c) and (d), unlike with the bifurcation diagrams shown in Fig. 5(b), (e) and (f), in the interval close to resonance frequency, the system exhibits periodic-1 motion with a relatively short interval. In addition, compared to normal system, the jump frequency increases under the effect of assembly error except assembly error $\Delta \varepsilon_{x}$. 

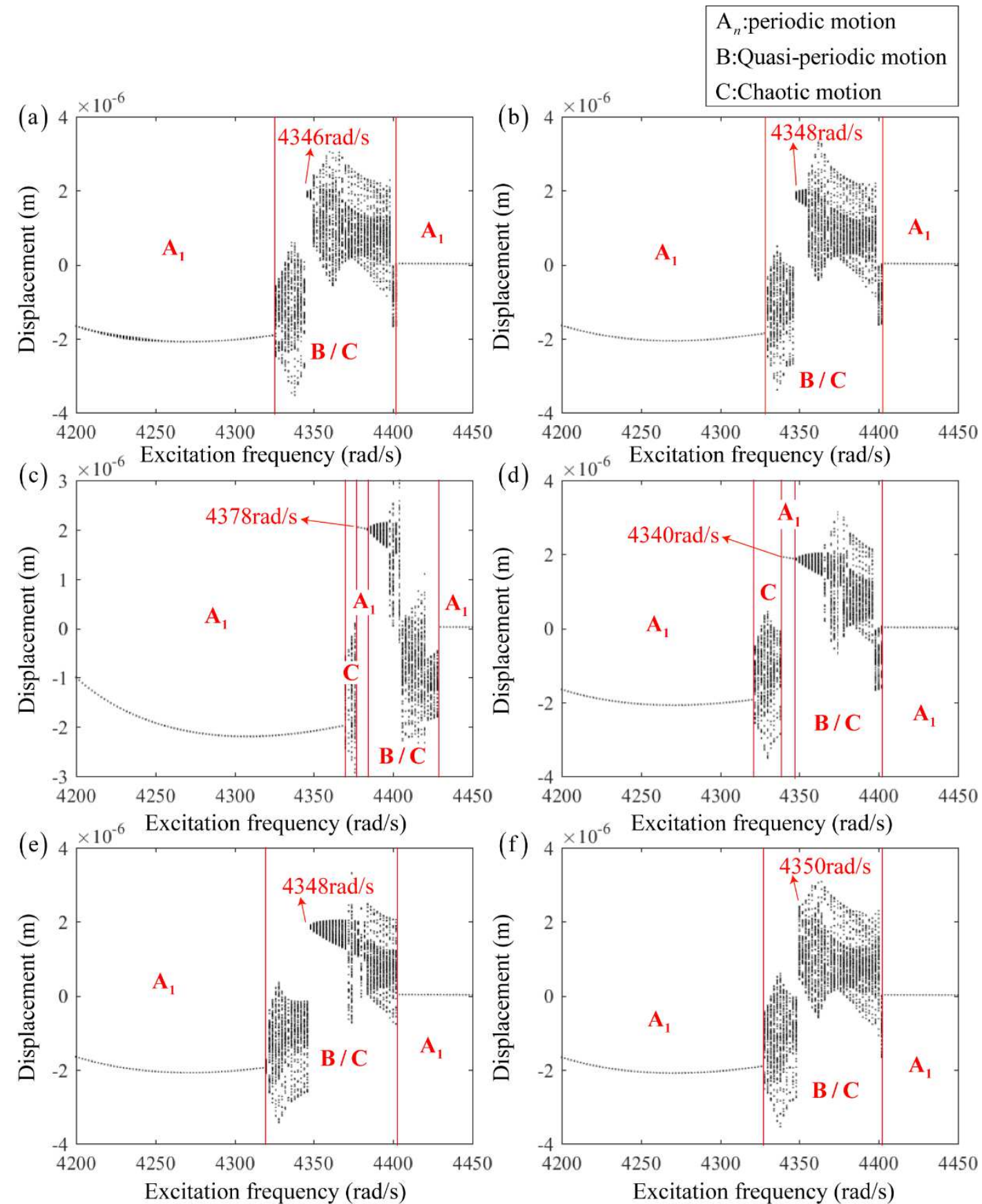

Fig. 5. Bifurcation diagrams with $\omega$ as control parameter. (a) Normal system, (b) with straightness assembly error $\Delta \delta_{x}^{\varepsilon}$, (c) with straightness assembly error $\Delta \delta_{y}^{\varepsilon}$ (d) with rotation assembly error $\Delta \varepsilon_{x}$, (e) with rotation assembly error $\Delta \varepsilon_{y}$, (f) with rotation assembly error $\Delta \varepsilon_{z}$. 


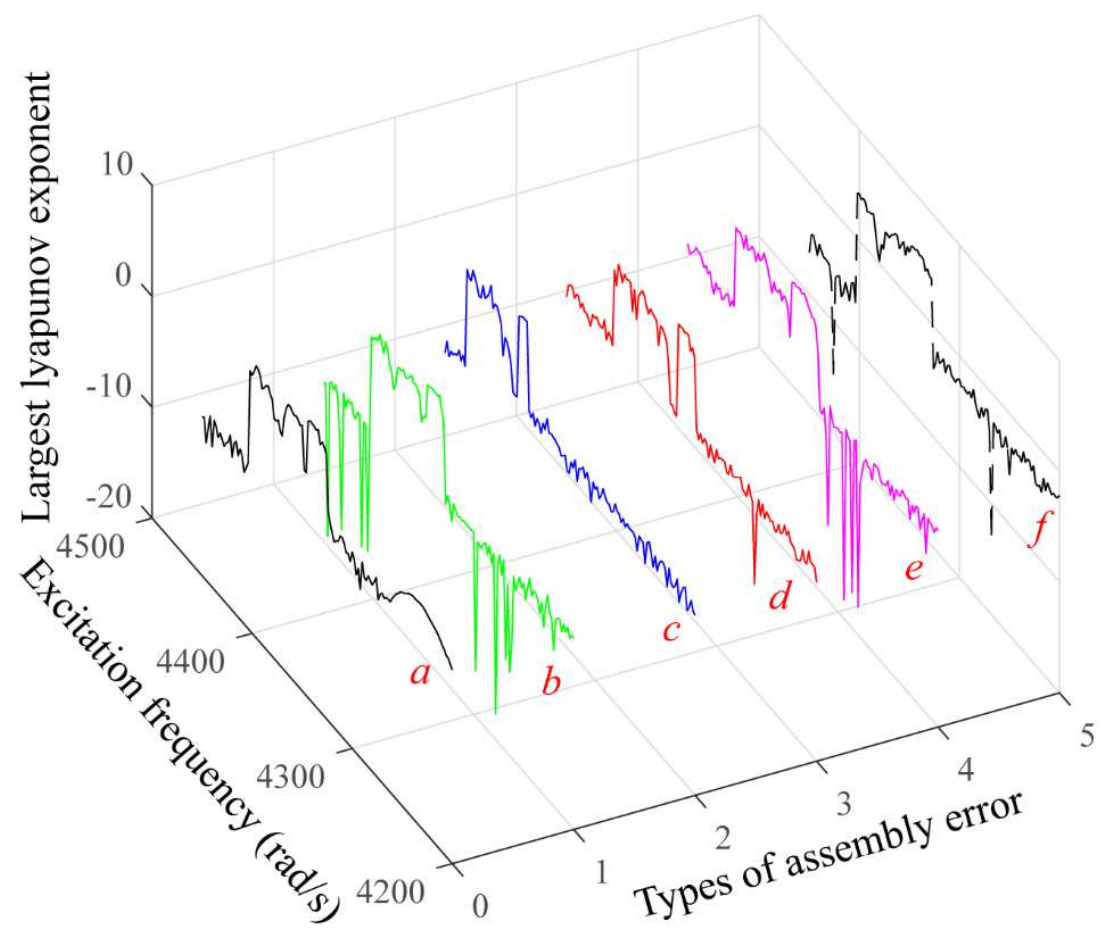

Fig. 6. 3-D LLE diagram with $\omega$ as control parameter. (a) Normal system, (b) straightness assembly error $\Delta \delta_{x}^{\varepsilon}$, (c) straightness error $\Delta \delta_{y}^{\varepsilon}$ (d) rotation error $\Delta \varepsilon_{x}$, (e)

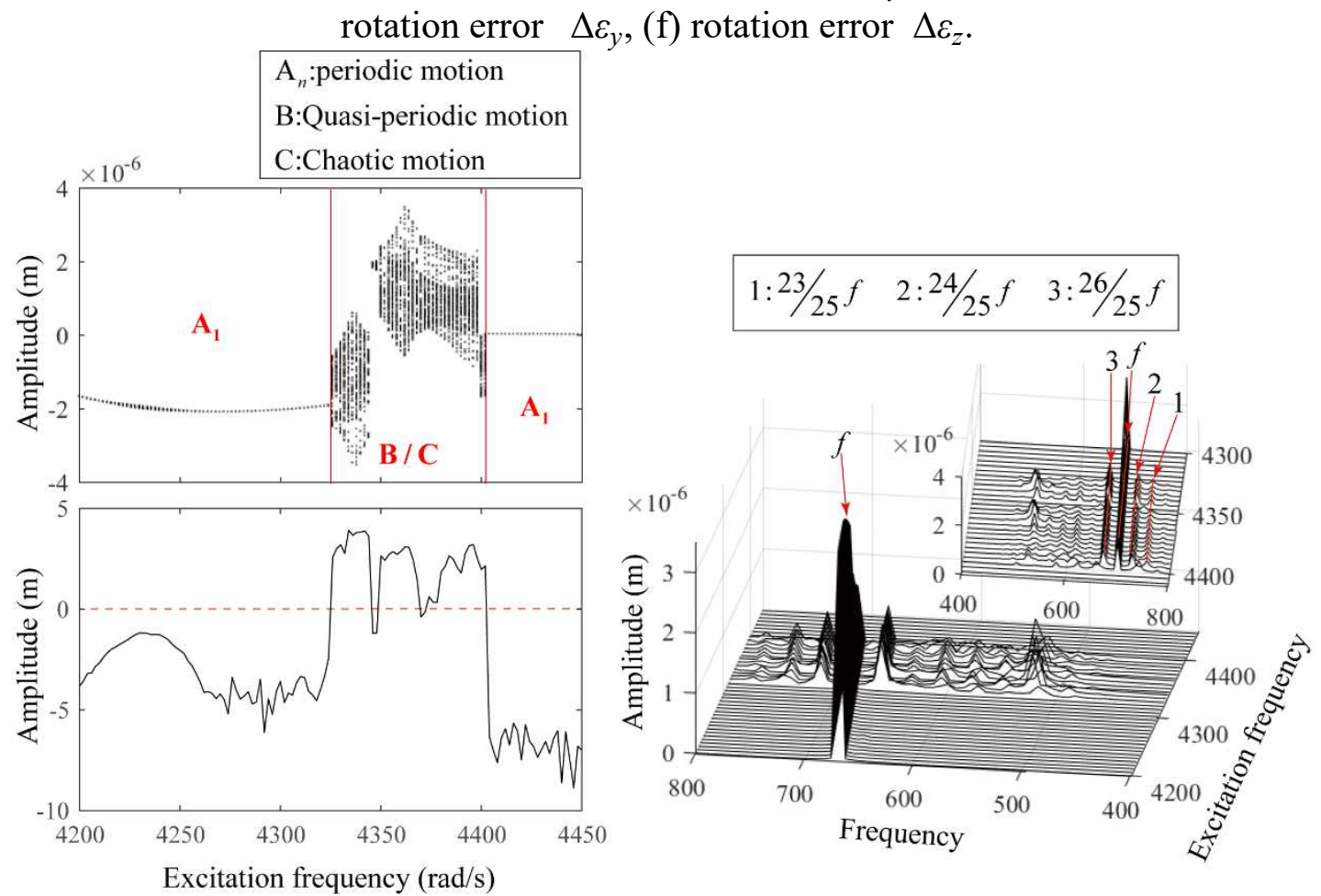

Fig. 7. Dynamic responses of the system without assembly error. (a) Bifurcation diagram, (b) LLE diagram, (b) 3-D frequency spectrum.

In order to illustrate the dynamic characteristics of linear guide slide platform in detail, the bifurcation diagram, LLE diagram, and 3-D frequency spectrum of the system without assembly error are shown in Fig. 7. In the second interval which is close to resonance frequency, the vibration system shows quasi-periodic and chaotic motions, frequency demultiplication appears $(23 / 25 f, 24 / 25 f, 26 / 25 f)$. Furthermore, the main frequency of the system is $f$. Fig. 8 displays the vibration response of the system without 
assembly error at $\omega=4280 \mathrm{rad} / \mathrm{s}$ and $\omega=4334 \mathrm{rad} / \mathrm{s}$. As shown in Fig. 8, which illustrates the motion of the system is periodic-1 motion. As can be seen in Fig. 9, frequency demultiplication components appear in Fig. 9(b), $f$ is the dominant frequency component, 19/25f is the second largest one, the Poincare section and phase diagram is regular which further illustrate the motion of the system is quasi-periodic motion at this point.

It can be concluded from the bifurcation diagram shown in Fig. 5(a)-(e). Under the action of different types of assembly error, the system can exhibit different nonlinear dynamic behaviors with respect to excitation frequency $\omega$. In addition, the assembly error can influence the frequency of jump discontinuity. By comparing the subfigures shown in Fig. 5, straightness assembly error $\Delta \delta_{x}^{\varepsilon}$, straightness assembly error $\Delta \delta_{y}^{\varepsilon}$, and rotation error assembly $\Delta \varepsilon_{y}$ can increase the jump frequency of the system. However, rotation error assembly $\Delta \varepsilon_{x}$ and rotation error assembly $\Delta \varepsilon_{z}$ decrease it.
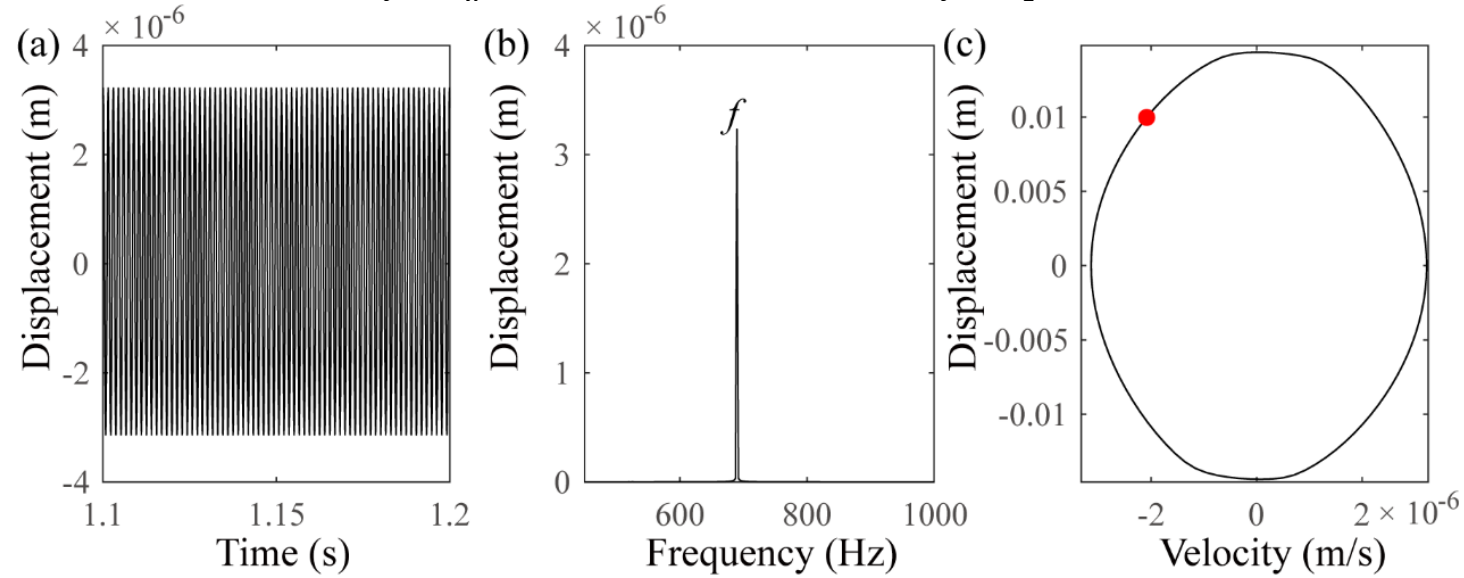

Fig. 8. Vibration responses at $\omega=4280 \mathrm{rad} / \mathrm{s}$. (a) Waveform diagram, (b) Spectrum, (c) Phase diagram and Poincaré section.

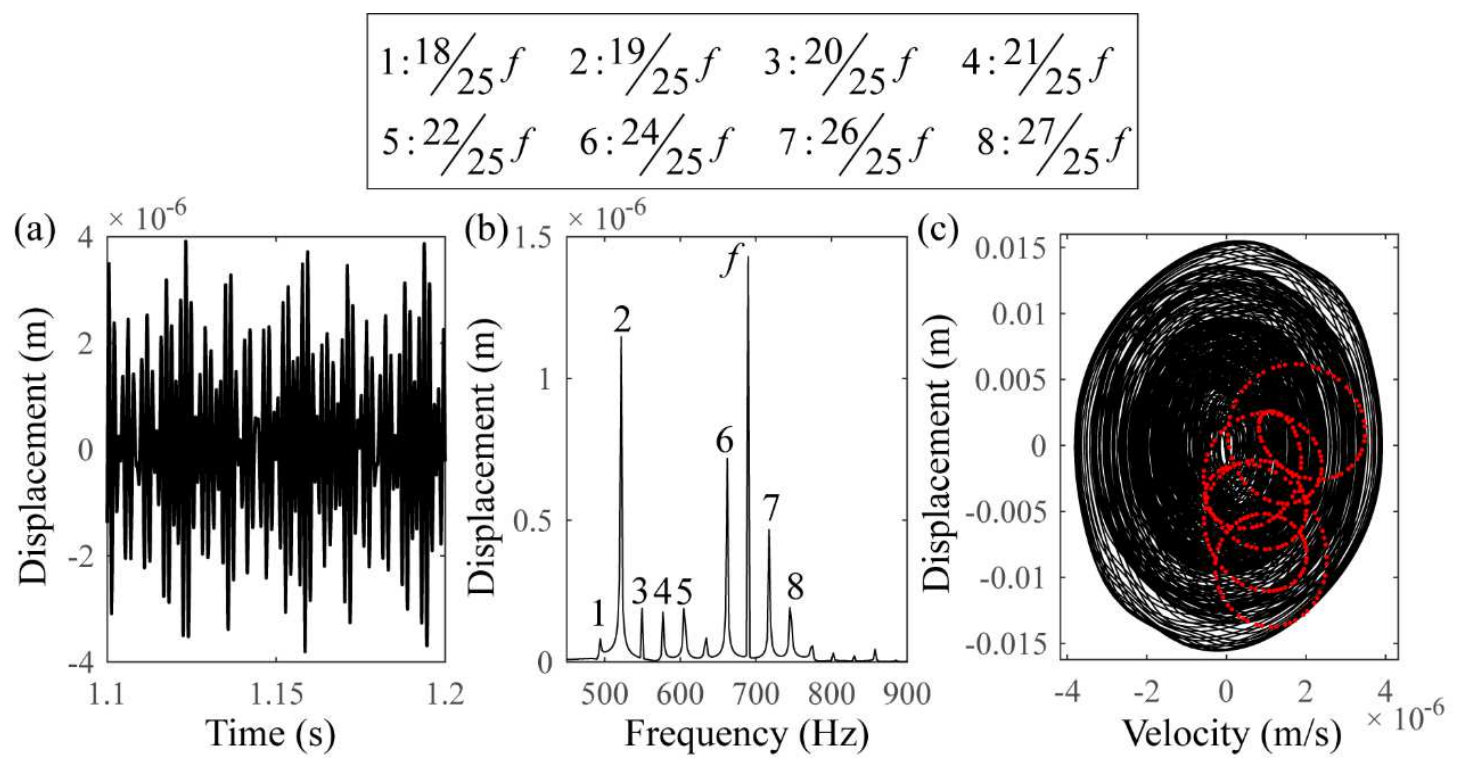

Fig. 9. Vibration responses at $\omega=4334 \mathrm{rad} / \mathrm{s}$. (a) Waveform diagram, (b) Spectrum, (c)

Phase diagram and Poincaré section.

\subsubsection{The influence of excitation amplitude}

Excitation amplitude is one of the most important factor that influence the dynamic behaviors of linear guide slide platform. In order to investigate the combined influence of assembly error and excitation amplitude, the bifurcation diagrams of the system with 
excitation amplitude $F_{0}$ as control parameter for different assembly errors are shown in Fig. 10. As shown in Fig. 10(a), (b), (d), (e) and (f), as the increase of excitation amplitude, the motion of the system from simply periodic- 1 motion in the first interval, to quasi-periodic motion in the second interval. The corresponding LLE diagram can be shown in Fig. 11(a), (b), (d), (e) and (f), the value of LLE first fluctuate intensively less than zero, and then increase up beyond zero. In Fig. 10(c), it is noteworthy that the system exhibits simply periodic- 1 motion in the whole range of $F_{0} \in[60000,100000] \mathrm{N}$. In addition, compare with normal system, jump discontinuity and quasi-periodic disappear.
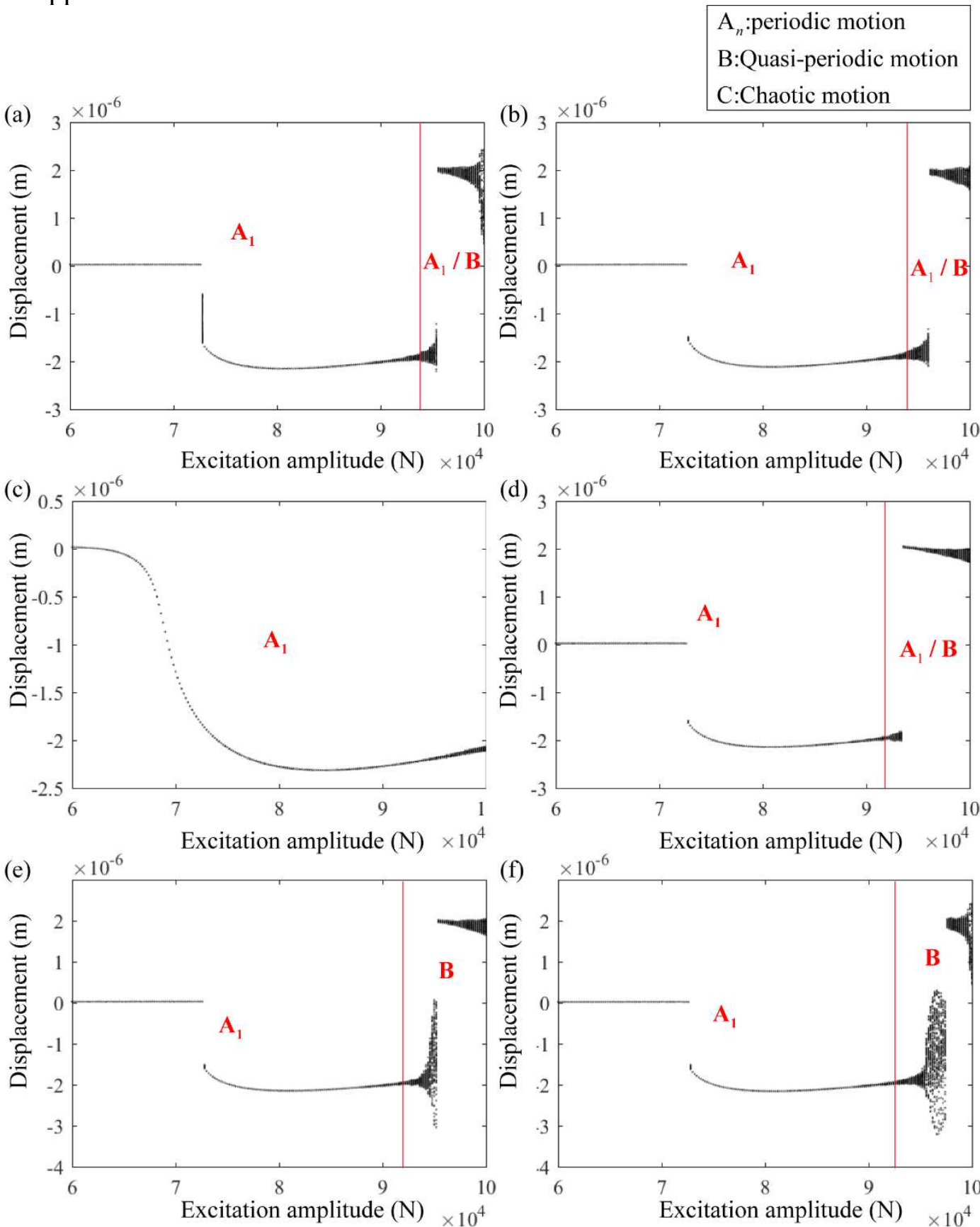

Fig. 10. Bifurcation diagrams with $F_{0}$ as control parameter. (a) Normal system, (b) with straightness assembly error $\Delta \delta_{x}^{\varepsilon}$, (c) with straightness assembly error $\Delta \delta_{y}^{\varepsilon}$ (d) with rotation assembly error $\Delta \varepsilon_{x}$, (e) with rotation assembly error $\Delta \varepsilon_{y}$, (f) with rotation assembly error $\Delta \varepsilon_{z}$. 


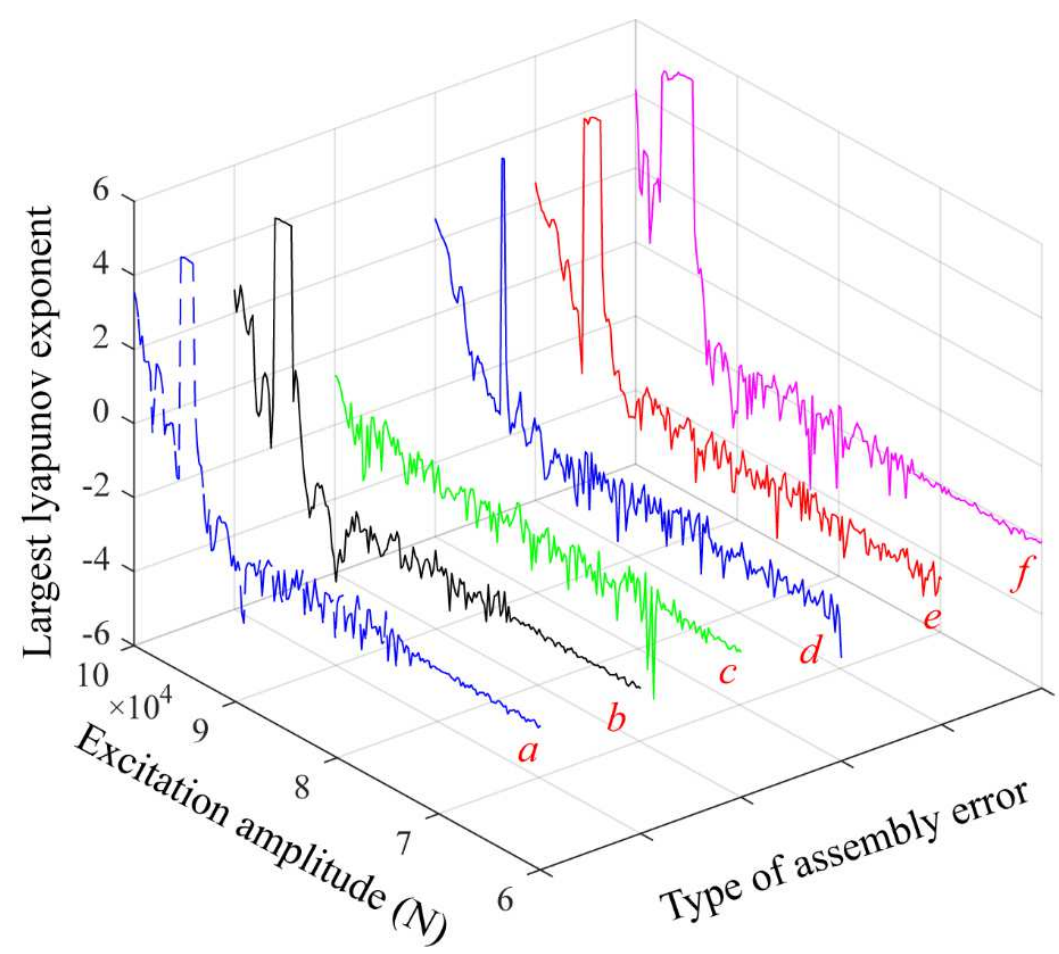

Fig. 11. 3-D LLE diagram for different types of assembly error. (a) Normal system, (b) straightness assembly error $\Delta \delta_{x}^{\varepsilon}$, (c) straightness assembly error $\Delta \delta_{y}^{\varepsilon}$ (d) rotation assembly error $\Delta \varepsilon_{x}$, (e) rotation assembly error $\Delta \varepsilon_{y}$, (f) rotation assembly error $\Delta \varepsilon_{z}$. In order to illustrate the influence of excitation amplitude on the dynamics of the system, Fig. 12 and Fig. 13 show the waveform, frequency, phase diagram and Poincaré section at $F_{0}=95800 \mathrm{~N}$ and $F_{0}=97400 \mathrm{~N}$ for assembly error $\Delta \varepsilon_{z}$, it can be observed that the frequency demultiplication components appear in the spectrum and the Poincaré section and Phase diagram are regular, which indicates that the system exhibit quasi-periodic motion at these two points.

$$
\begin{array}{|lll|}
1: 18 / 25^{f} & 2: 19 / 25^{f} & 3: 22 / 25^{f} \\
4: 24 / 25^{f} & 5: 26 / 25^{f} & 6: 27 / 25^{f} \\
\hline
\end{array}
$$
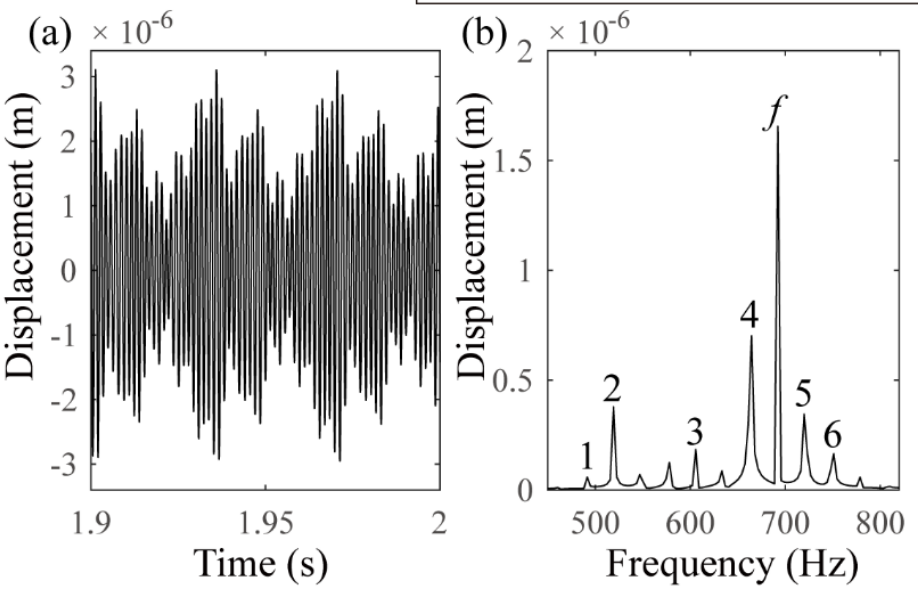

(c)

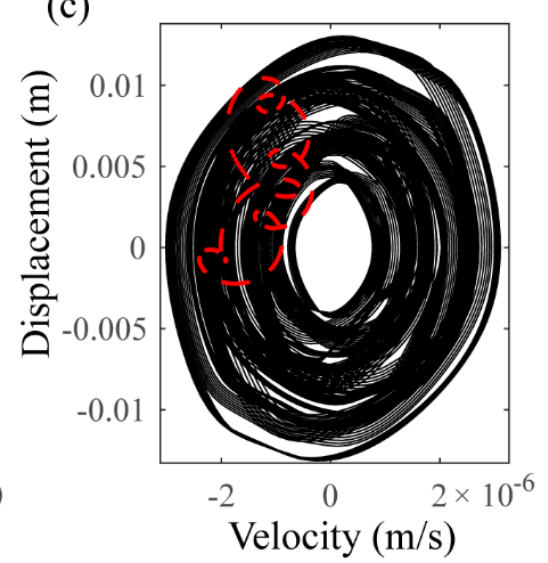

Fig. 12. Vibration responses at $F_{0}=95800$ N. (a) Waveform diagram, (b) Spectrum, (c)

Phase diagram and Poincaré section. 


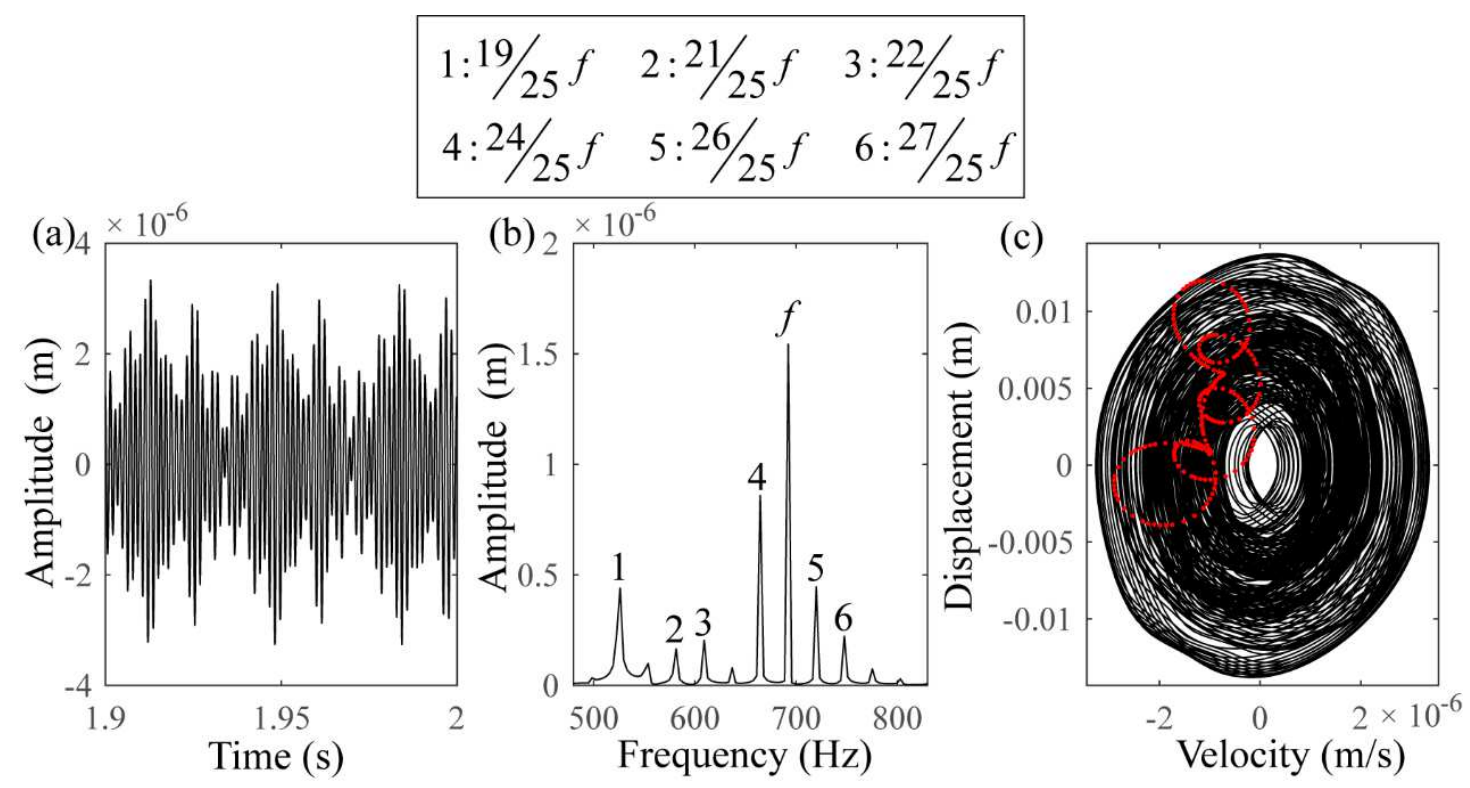

Fig. 13. Vibration responses at $F_{0}=97400$ N. (a) Waveform diagram, (b) Spectrum, (c)

Phase diagram and Poincaré section.

In conclusion, the excitation amplitude is a sensitive parameter, which influence the motion state of the system and lead the system to take on relatively strong nonlinearity and instability. Furthermore, the combined effect of the assembly error and excitation amplitude make the motion of the system complex.

\subsubsection{The influence of assembly error}

In this section, the dynamic characteristics of the system are investigated with five types of assembly error as control parameter. As can be seen in Fig.14 (a) and (b), there exist two intervals, according to the corresponding LLE diagram shown in Fig. 15, the system exhibits quasi-periodic motion and chaotic motion in the first interval. As $\Delta \delta_{x}^{\varepsilon}$ and $\Delta \delta_{y}^{\varepsilon}$ are increased, the values of LLE are less than zero, and therefore, the nature of the motions of the system are periodic-1. As it can be seen in Fig. 14(c), (d) and (e), the system exhibits predominantly chaotic and quasi-periodic motion in the whole assembly error range, and the value of LLE fluctuates intensively in Fig. 15. For assembly error $\Delta \varepsilon_{z}$, with the increase of $\Delta \varepsilon_{z}$ from low to high, the nature of the motion are chaotic and quasi-periodic motion. In Fig. 14(e), it is noteworthy that the nature of the system does not change with assembly error $\Delta \varepsilon_{z}$.

To investigate the dynamic characteristic of the system, Fig. 16 shows the bifurcation diagram, LLE diagram, and 3-D spectrum with assembly error $\Delta \delta_{y}^{\varepsilon}$ as control parameter. As shown in the 3-D spectrum, in the first interval, the main frequency component is $f$, the demultiplication frequency component appears, as the increase of assembly error $\Delta \delta_{y}^{\varepsilon}$, the demultiplication frequency component disappear in the second interval. 

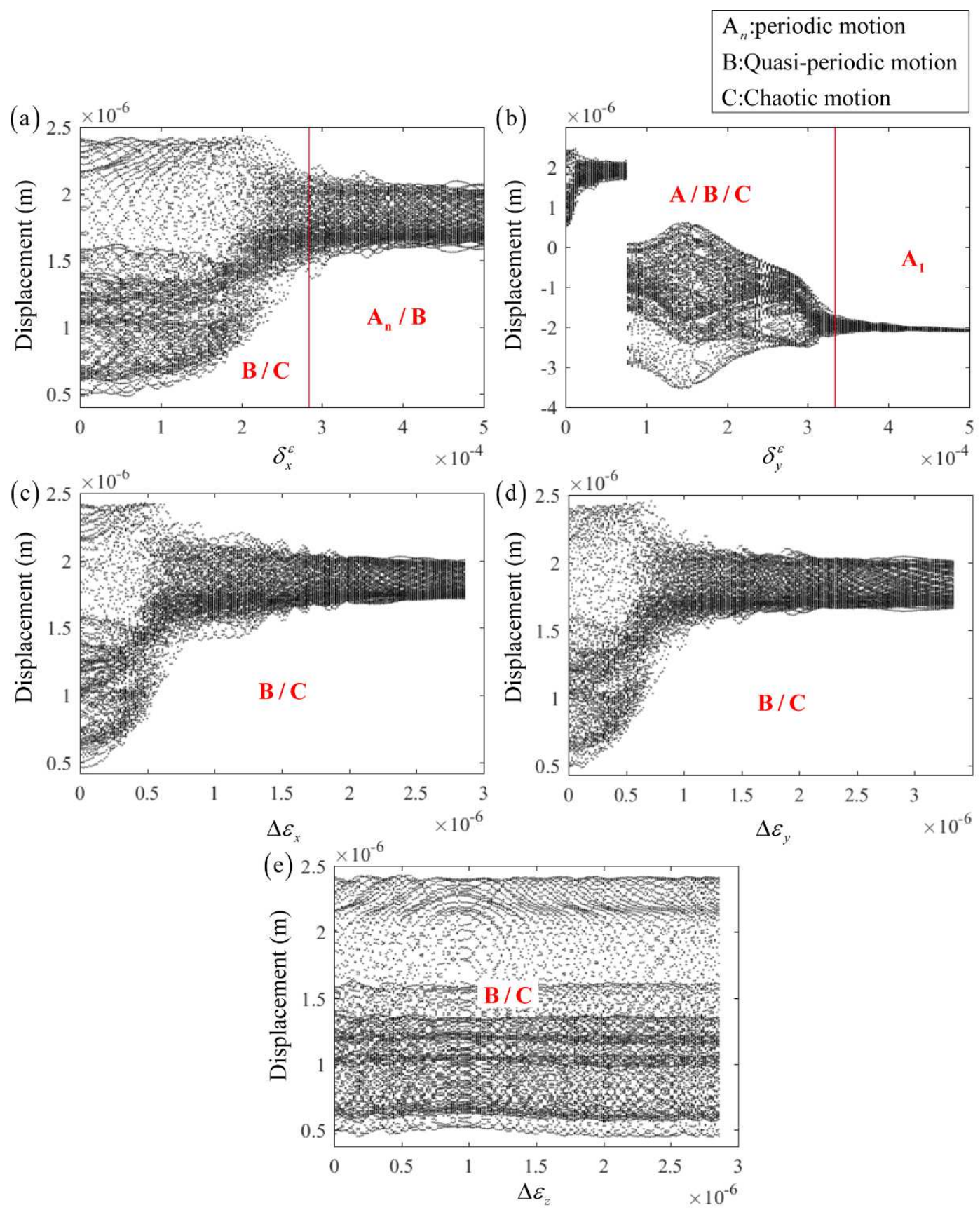

Fig. 14. Bifurcation diagram with different assembly errors as control parameter. (a) straightness assembly error $\Delta \delta_{x}^{\varepsilon}$, (b) straightness assembly error $\Delta \delta_{y}^{\varepsilon}$ (c) rotation assembly error $\Delta \varepsilon_{x}$, (d) rotation assembly error $\Delta \varepsilon_{y}$, (e) rotation assembly error $\Delta \varepsilon_{z}$. 


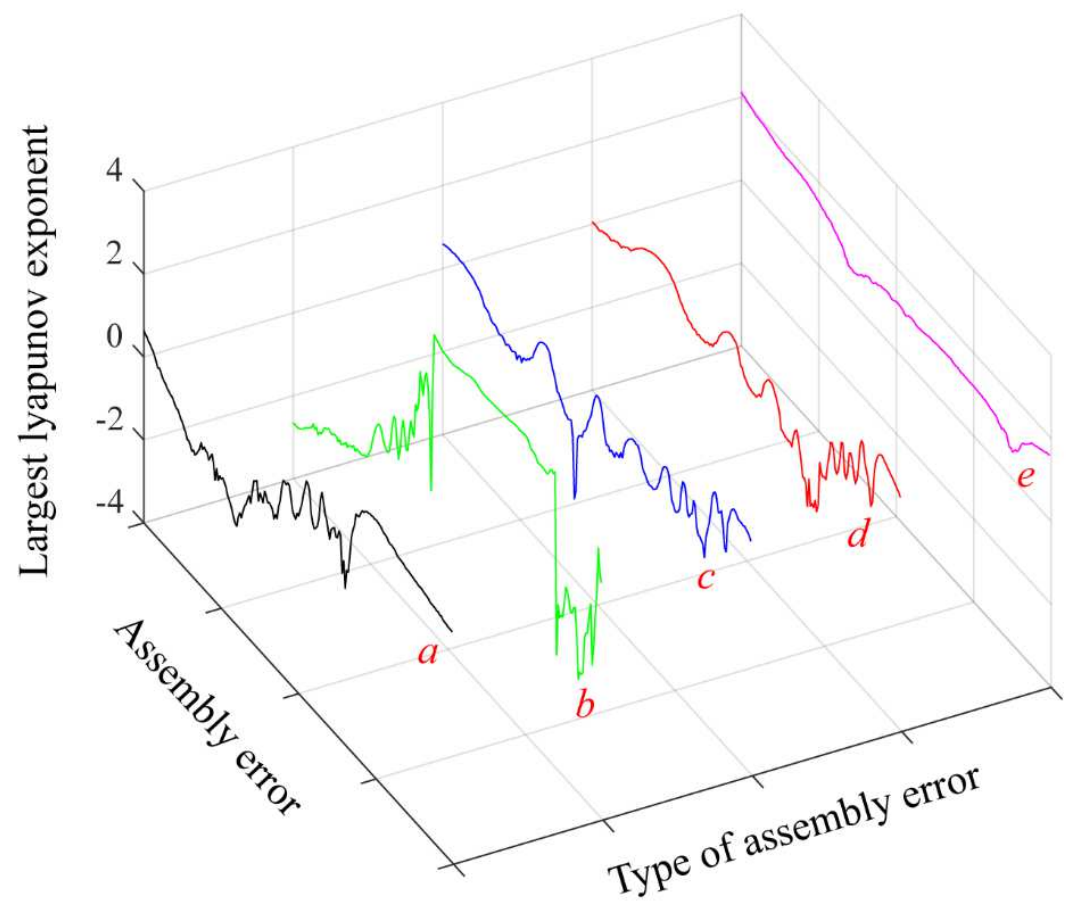

Fig. 15. 3-D LLE diagram with different types of assembly error as control parameter. (a) straightness assembly error $\Delta \delta_{x}^{\varepsilon}$, (b) straightness assembly error $\Delta \delta_{y}^{\varepsilon}$ (c) rotation assembly error $\Delta \varepsilon_{x}$, (d) rotation assembly error $\Delta \varepsilon_{y}$, (e) rotation assembly error $\Delta \varepsilon_{z}$.
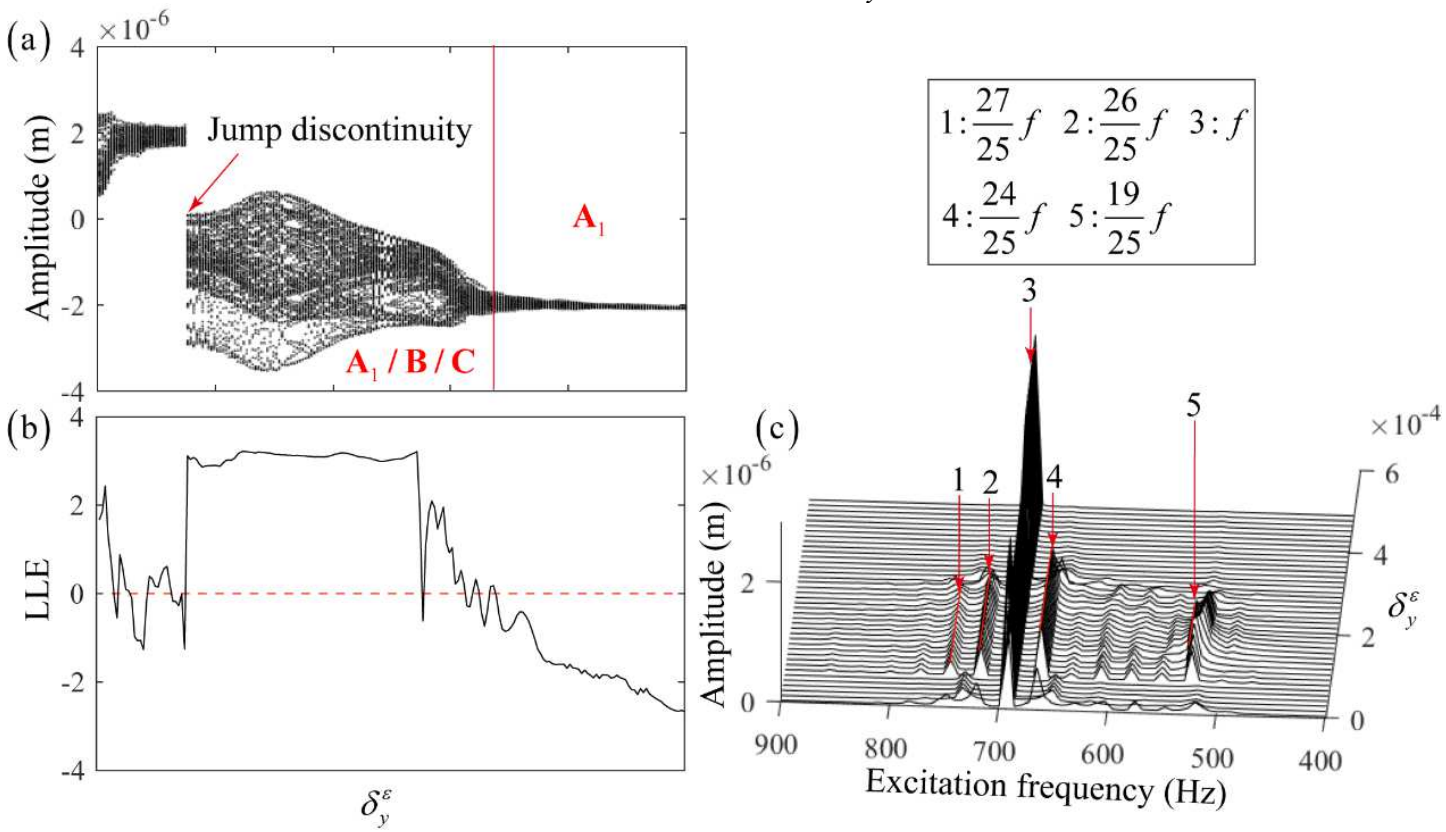

Fig. 16. Dynamic responses with $\Delta \delta_{y}^{\varepsilon}$ as control parameter. (a) Bifurcation diagram,

\subsubsection{The influence of preload}

(b) LLE diagram, (b) 3-D frequency spectrum.

In order to investigate the combined influence of preload and assembly error on the dynamic characteristics of the system, Fig. 17 and Fig. 18 present the bifurcation diagram and LLE diagram with preload as control parameter in the interval $[0.9,1.15]$ $F_{p}$. It can be seen in Fig. 17 (a), (b), (c) and (f), there exist three intervals in the bifurcation diagrams, by increasing the preload, the motion of the system from simple 
periodic-1 motion in the first interval, through chaotic and quasi-periodic motion in the second interval, the jump discontinuity phenomenon can be observed in this interval, at last the system enters to periodic- 1 and quasi-periodic motion in the third interval. It can be observed that the stability of the system do not improved with the increase of the preload of the system. As shown in Fig. 17(d) and (e), the system experienced a short range of quasi-periodic motion in the third interval. In addition, in Fig. 17(c), compared with other bifurcations, the preload entering into chaotic motion is less than other cases. The dynamic response of the system with assembly error $\Delta \varepsilon_{z}$ can be seen in Fig. 19, the continuous frequency components appear in the 3-D frequency spectrum, which indicates the system exhibits chaotic motion in the corresponding interval.
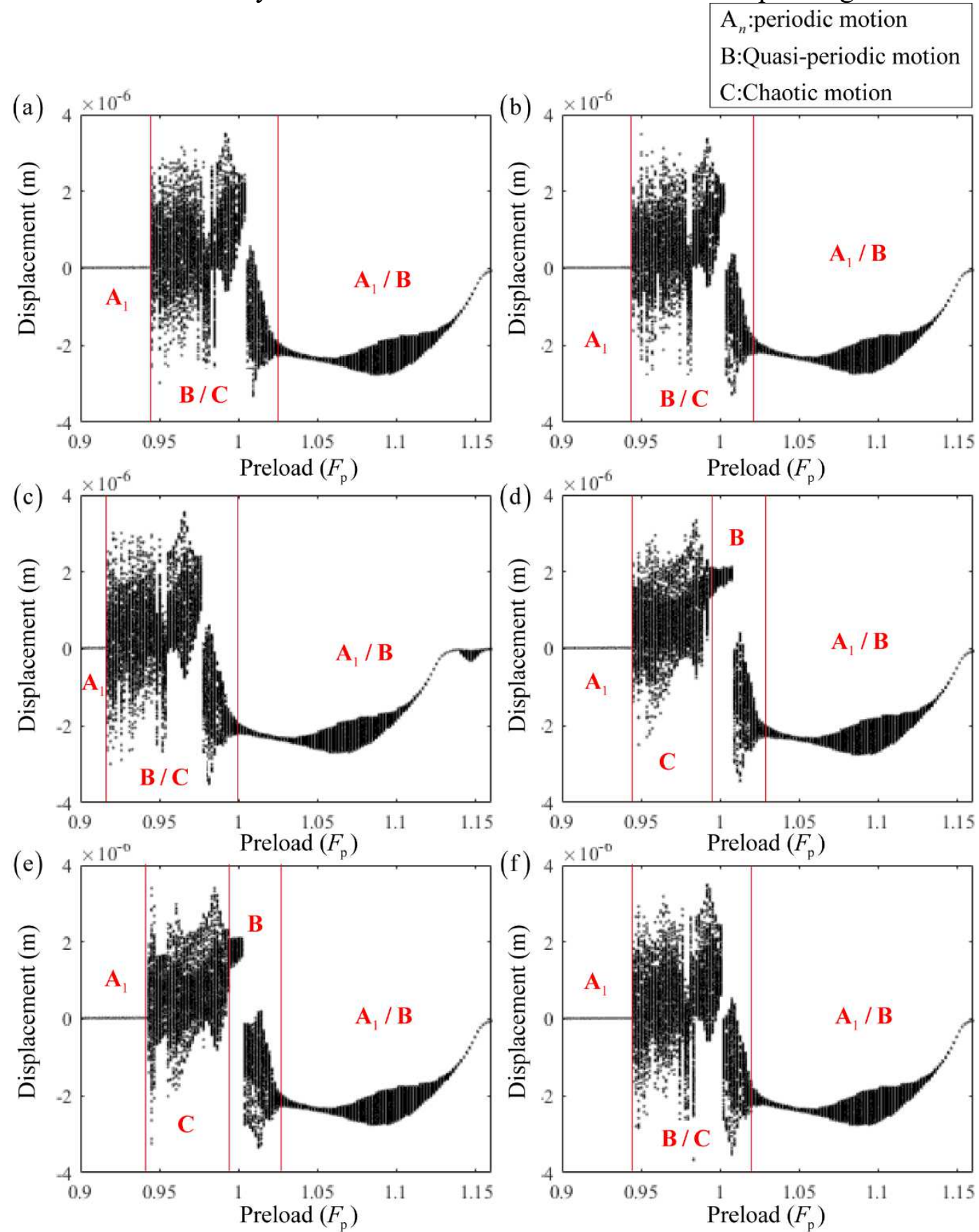

Fig. 17. Bifurcation diagram with preload as control parameter. (a) Normal system. (b) straightness assembly error $\Delta \delta_{x}^{\varepsilon}$, (c) straightness assembly error $\Delta \delta_{y}^{\varepsilon}$ (d) rotation assembly error $\Delta \varepsilon_{x}$, (e) rotation assembly error $\Delta \varepsilon_{y}$, (f) rotation assembly error $\Delta \varepsilon_{z}$. 
The vibration response of the system at preload $=0.9832 F_{\mathrm{p}}$ can be shown in Fig. 20 , there exist numerous points in Poincaré section, continuous frequency components appear, waveform diagram and phase diagram are disorder, which illustrates that the system exhibits chaotic motion at this point. The vibration response of the system at preload $=0.9555 F_{\mathrm{p}}$ is shown in Fig. 21 , frequency demultiplication $(39 f / 54,41 f / 54$, $52 f / 54,56 f / 54,58 f / 54)$ components appear, Poincaré section and phase diagram are regular, which indicates that the system displays quasi-periodic motion.

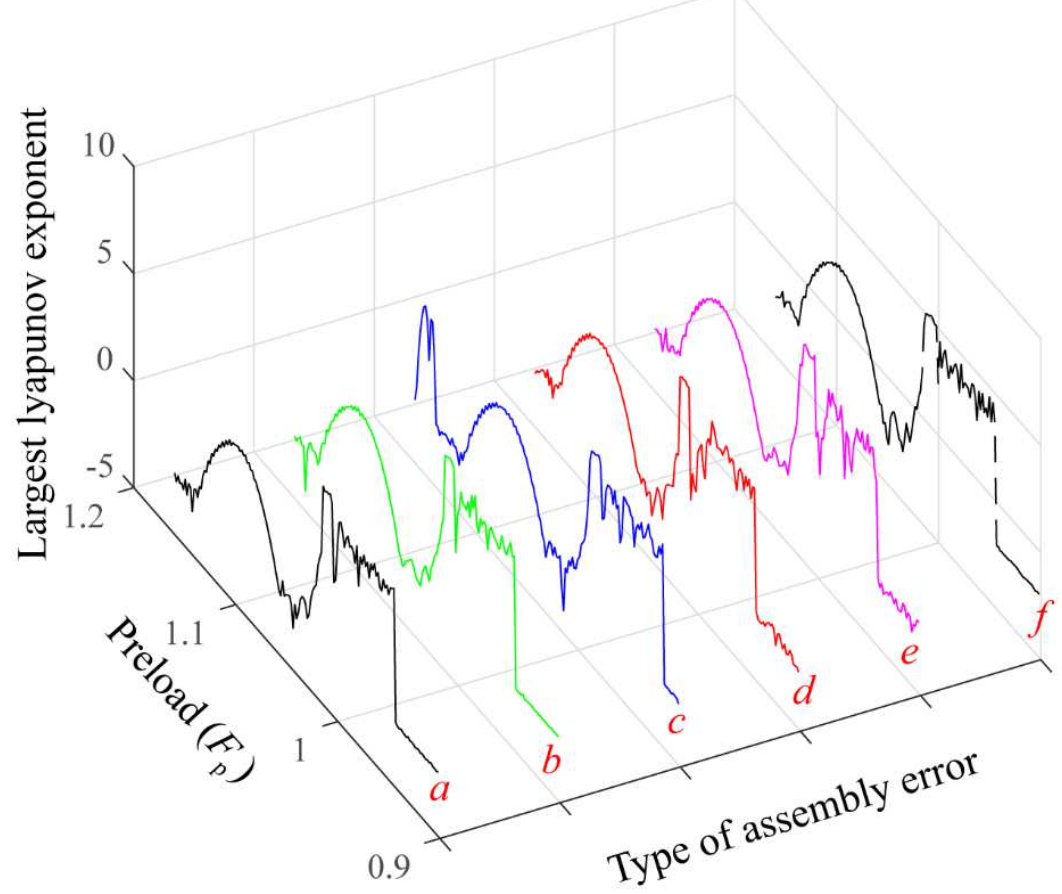

Fig. 18. 3-D LLE diagram with preload as control parameter. (a) straightness assembly error $\Delta \delta_{x}^{\varepsilon}$, (b) straightness error $\Delta \delta_{y}^{\varepsilon}$ (c) rotation error $\Delta \varepsilon_{x}$, (d) rotation error $\Delta \varepsilon_{y},(\mathrm{e})$ rotation error $\Delta \varepsilon_{z}$.
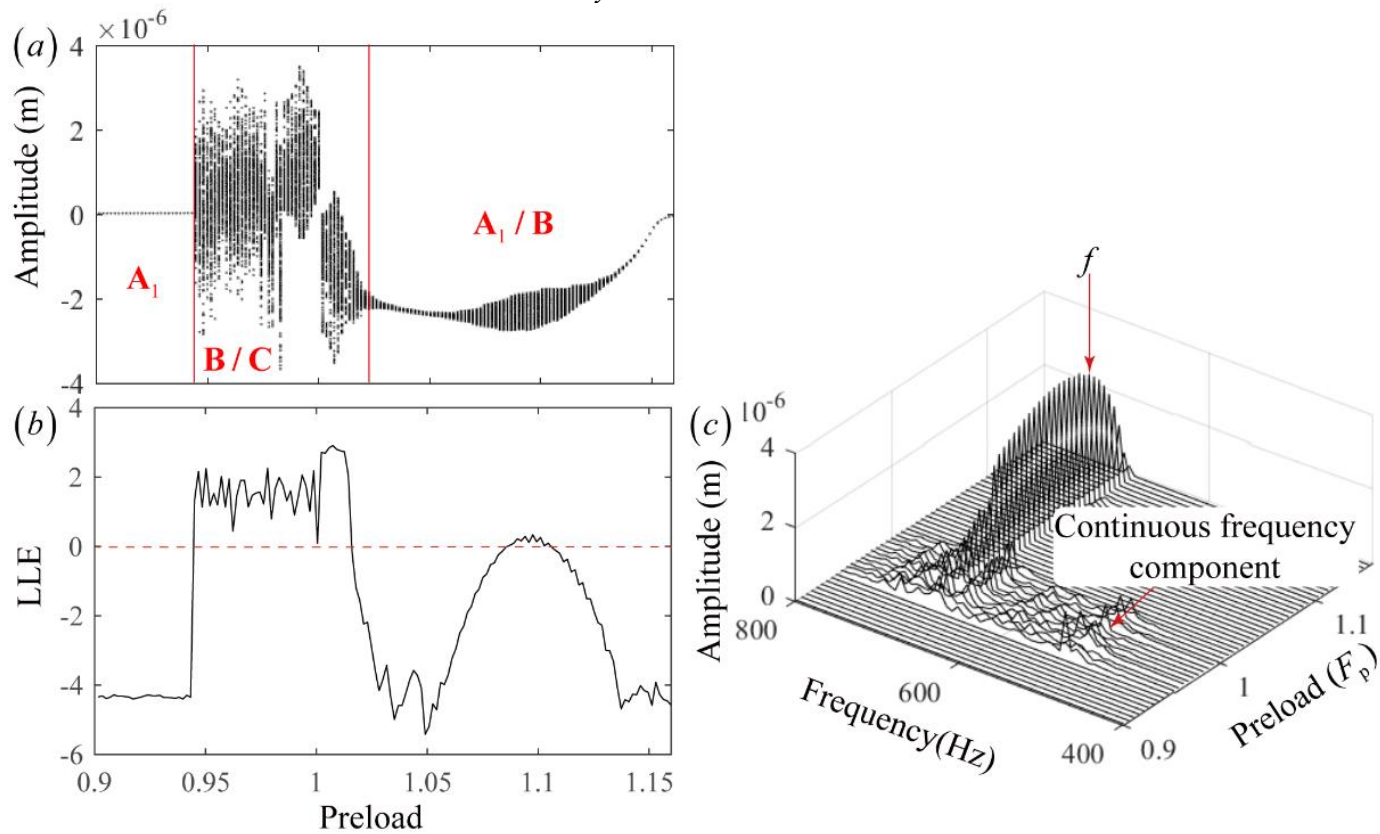

Fig. 19. Dynamic responses with preload as control parameter for assembly error $\Delta \varepsilon_{z}$. (a) Bifurcation diagram, (b) LLE diagram, (b) 3-D frequency spectrum. 


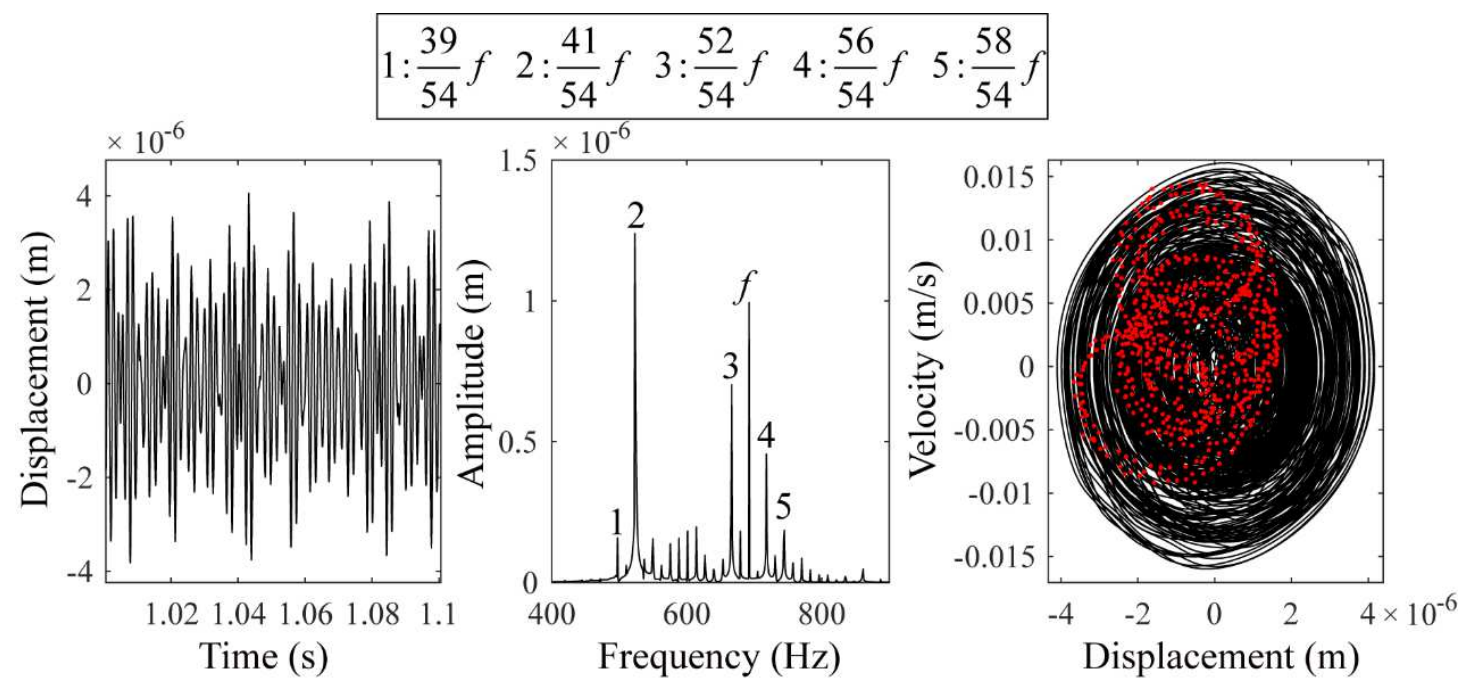

Fig. 20. Vibration responses at preload $=0.9832 F_{\mathrm{p}}$. (a) Waveform diagram, (b)

Spectrum, (c) Phase diagram and Poincaré section.

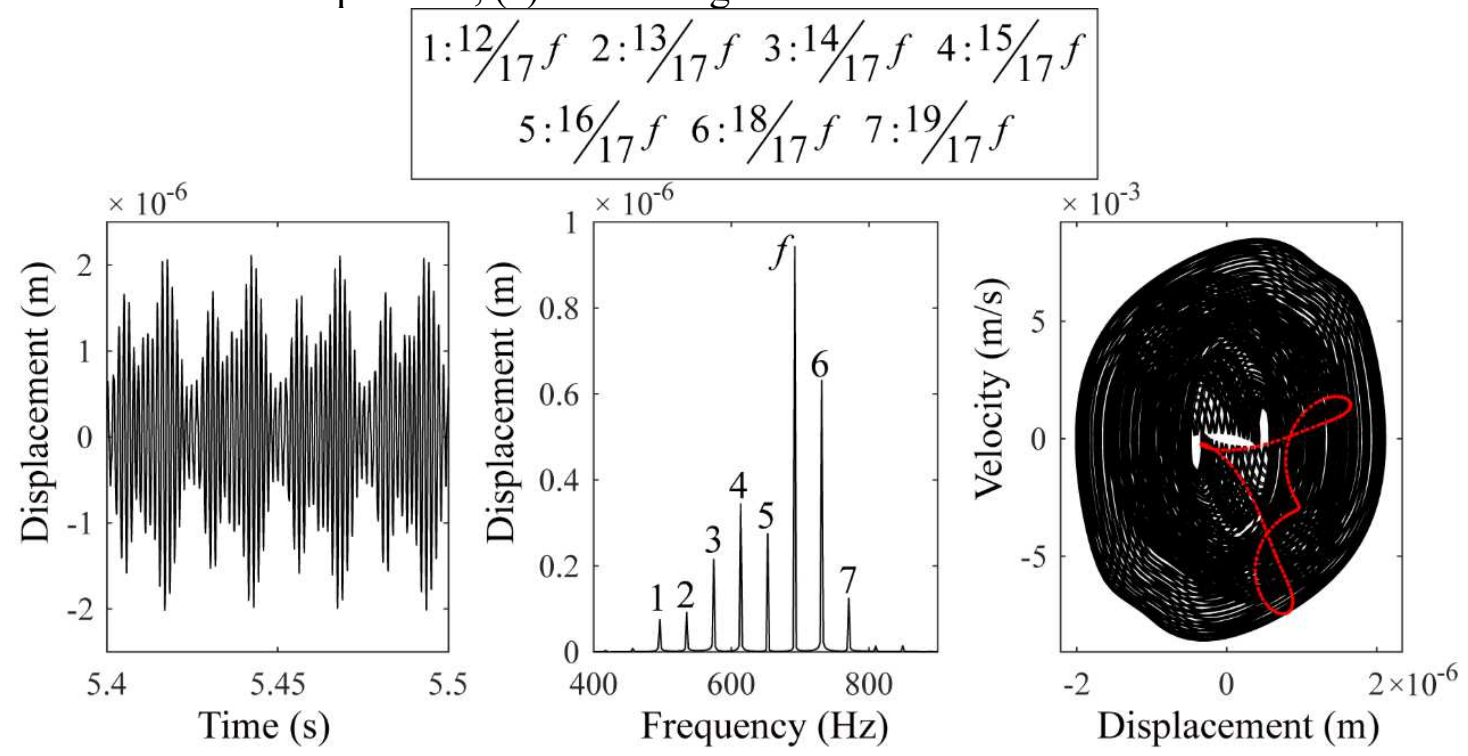

Fig. 21. Vibration responses at preload $=0.9555 F_{\mathrm{p}}$. (a) Waveform diagram, (b)

Spectrum, (c) Phase diagram and Poincaré section.

\section{Model verification and system parameter estimation}

In this section, a series of tests are conducted on a specialized linear guideway slide platform to validate the proposed dynamic model and estimate the system parameters.

\subsection{System parameter estimation}

Damping ratio determines the amplitude of vibration response at resonance frequency, and therefore, the estimation of damping ratio is critical for the design and investigation of the system [33]. In order to estimate the damping ratio of the system, an impact hammer test is conducted, and the test setup is shown in Fig. 22. As shown in the figure, an impact hammer (Sinocera LC-01A) is used to excite a range of frequencies above the noise floor of the instrument, the output of the system is measured by an accelerometer (Piezotronics 352C04), and processed by a data collection system(DongHua5956) and a PC. The frequency response function (FRF) can be obtained by:

$$
\mathrm{FRF}=\frac{\text { output }}{\text { input }}
$$




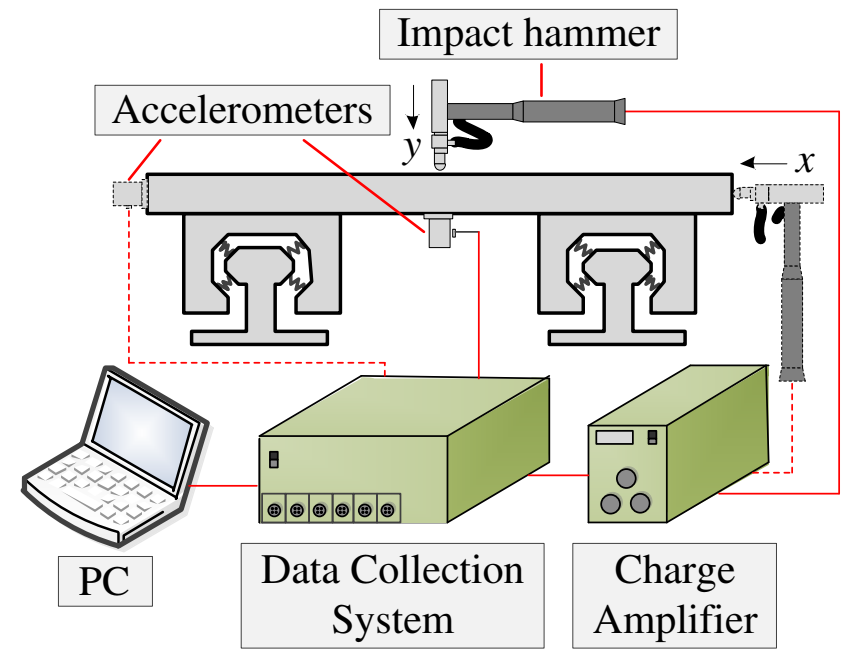

Fig. 22 schematic of impact hammer test.

According to the equation in Ref. [34], the expression of damping ratio $\zeta$ can be given by:

$$
\zeta=\frac{\omega_{2}-\omega_{1}}{2 \omega_{m}}
$$

where $\omega_{1}$ and $\omega_{2}$ represent the half-power frequency when the corresponding amplitude $A_{1}$ and $A_{2}$ are equal to $A_{\max } / \sqrt{2}$, and $\omega_{m}$ is the resonance frequency. The FRF of the system along $x$ and $y$ axis can be shown in Fig. 23, $\omega_{x 1}=1683 \mathrm{~Hz}, \omega_{x 2}=1784 \mathrm{~Hz}, \omega_{y 1}=$ $687 \mathrm{~Hz}, \omega_{y 2}=706.4 \mathrm{~Hz}$, and the damping ratio along $x$ and $y$ axis can be estimated $\zeta_{x}$ $=0.0297$ and $\zeta_{y}=0.0139$. Therefore, the viscous damping coefficient along $x$ and $y$ directions can be estimated by $c=4 \pi \zeta \omega_{n} m$ respectively.
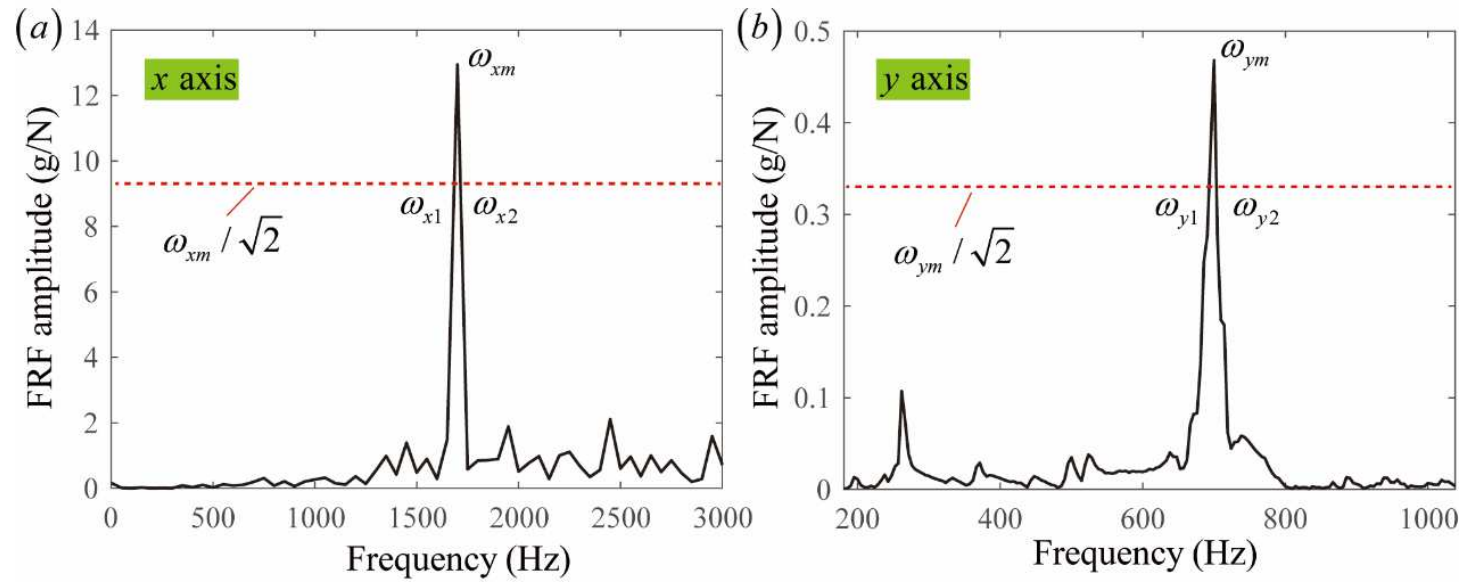

Fig. 23 Impact hammer test results. (a) frequency response function along $x$ axis, (b)

\subsection{Experimental verification}

frequency response function along $y$ axis.

In this section, a test is conducted on a specialized linear guide worktable to measure the vibration response of the system, which is used to compare the vibration response obtained by the model. The schematic of the test system can be shown in Fig. 24 , the test system is composed of two subsystem, namely, data collection system and excitation system. The descriptions of the two system are as follows, the exciting force applied on the worktable is generated by an electromagnetic shaker (Sinocera JZK- 50), the excitation amplitude and excitation frequency of the force are controlled by a signal generator (Sinocera YE1311) and a power amplifier (YE5874A), the excitation amplitude is measured by a piezoelectric force senor (Sinocera CL-YD-331A). The vibration response of the system is measured by an accelerometer (Piezotronics 352C04) 
from five directions, a data collection system (DH5956) and a PC are used to process and save data. In this experiment, using a gasket to simulate rotation error $\Delta \varepsilon_{x}$. The thickness of the gasket is $4 \mathrm{~mm}, \mathrm{~L}=150 \mathrm{~cm}$, and therefore, the rotation error of Rail II is $\Delta \varepsilon_{x}=0.0053$. The comparisons of dynamic response between simulation and experiment are shown in Fig. 25.

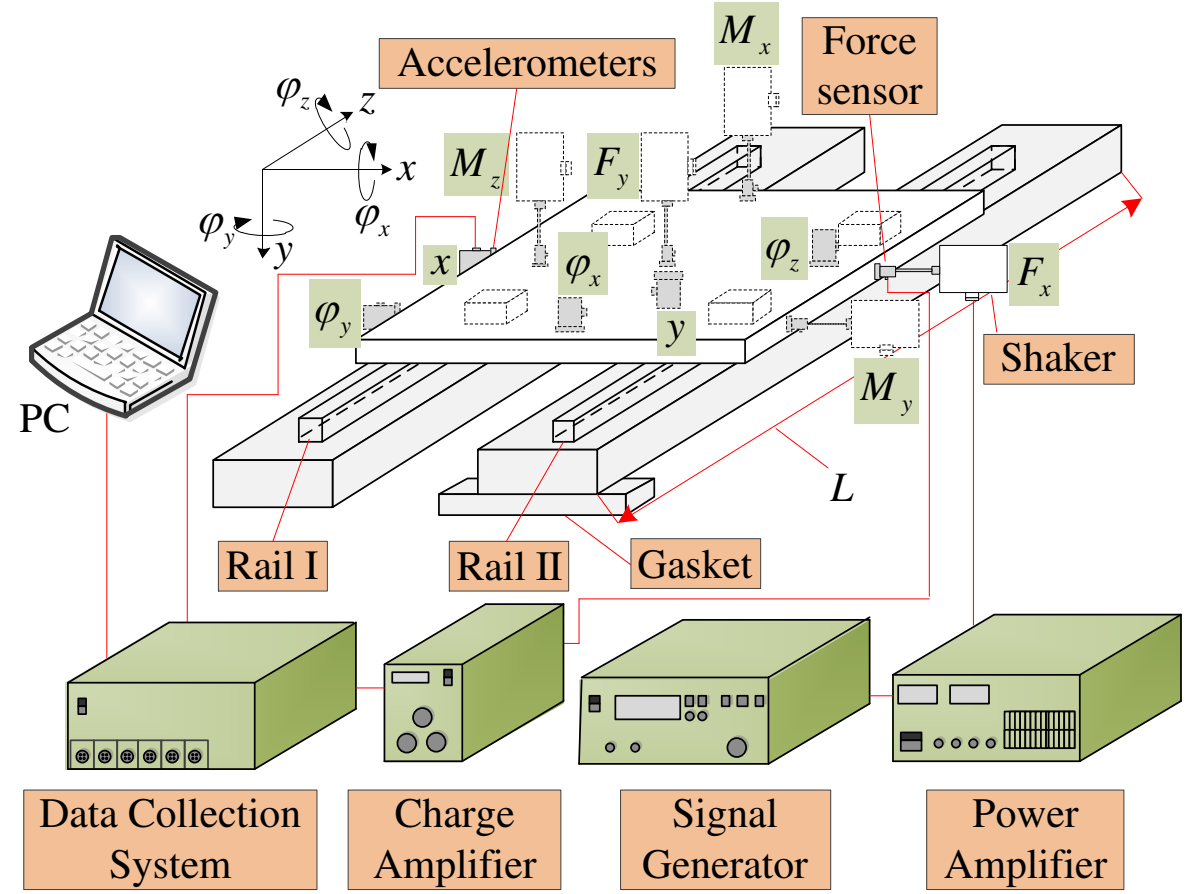

Fig. 24 Schematic diagram of vibration test. 


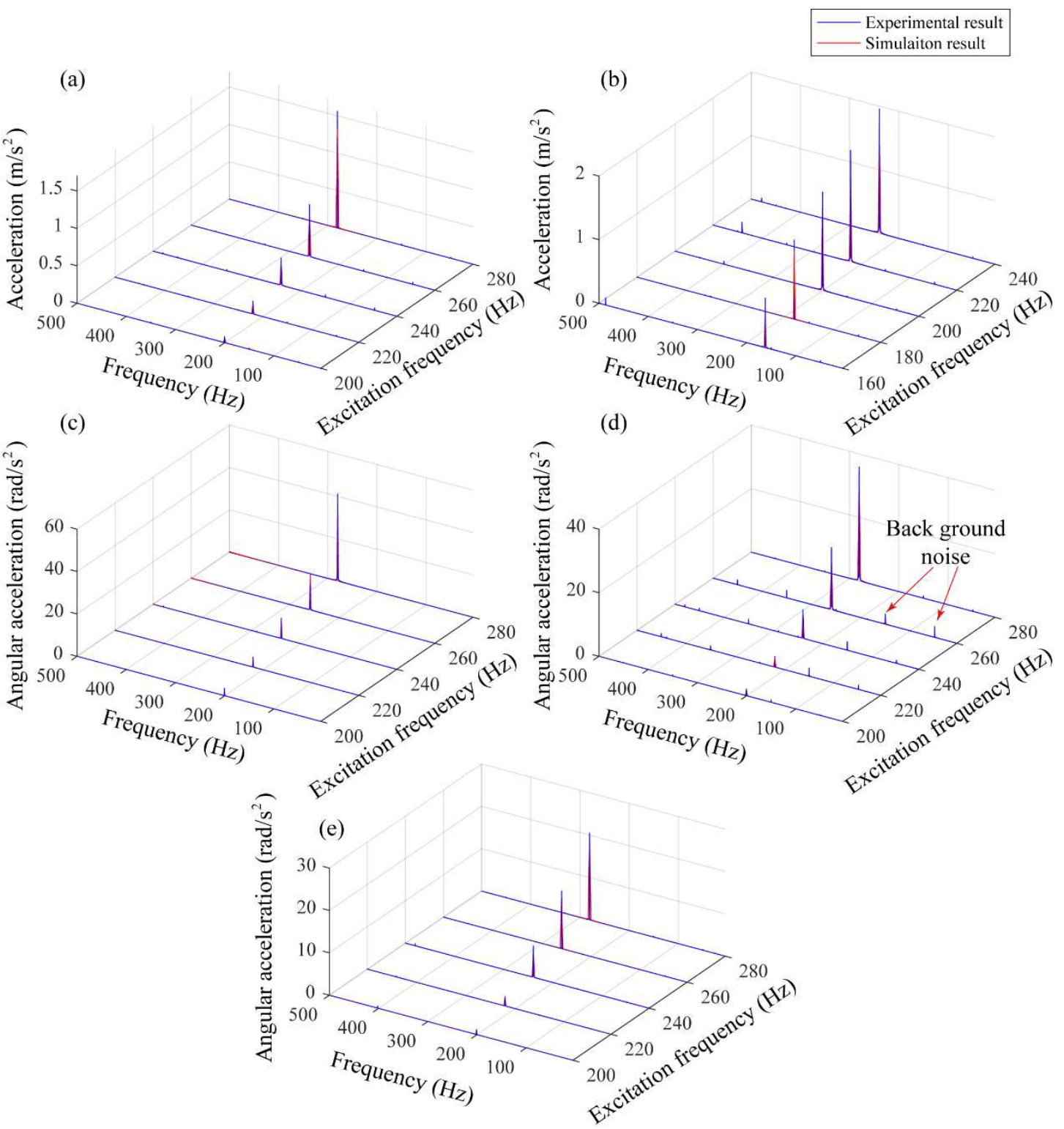

Fig. 25. Comparisons of vibration response between simulation and experiment. (a) along $x$ axis, (b) along $y$ axis, (c) about $x$ axis, (d) about $y$ axis, (e) about $y$ axis.

\section{Conclusion}

In this study, a new 5-degrees-of-freedom nonlinear dynamic model of linear guide slide platform is developed, and five types of assembly errors including two straightness errors and three rotational errors are introduced into the nonlinear dynamic model. The influence of the assembly error on load distribution and positioning accuracy is analyzed. The combined effect of system parameters and each type of assembly error on the dynamic characteristic of the system is studied. A series of experiment are conducted to validate the proposed method. Some conclusions of the study can be obtained as follows: All five types of assembly errors can cause uneven load distribution between grooves, and rotation error $\Delta \varepsilon_{x}$ and $\Delta \varepsilon_{y}$ can lead to uneven load distribution in a groove. Assembly error can influence the dynamic characteristic of the linear guide slide platform, change the frequency of jump discontinuity and resonance frequency. Excitation amplitude can have impact to the system, and the system with straightness assembly error $\Delta \varepsilon_{z}$ is less sensitive to excitation amplitude. The stability of the system cannot be improved by simply increasing the preload. 


\section{Acknowledgment}

The work was supported by National Natural Science Foundation of China (Grant No. 52075087), the Fundamental Research Funds for the Central Universities (Grant No. N2003006, and N2103003), and the Natural Science Foundation of Heilongjiang Province (HL2020A017).

\section{Declarations}

\section{Funding}

The work was supported by National Natural Science Foundation of China 524(Grant No. 52075087), the Fundamental Research Funds for the Central Universities(Grant No. N2003006 and N2103003), National Natural Science Foundation of China(Grant No. U1708254)

\section{Conflicts of interest}

The authors declare that they have no known competing financial interests or personal relationships that could have appeared to influence the work reported in this paper.

\section{Availability of data and material}

The data sets supporting the results of this article are included within the article and its additional files.

Authors' contributions

Zhendong Liu: Methodology; Investigation; Experimental; Writing-Original Draft; Writing- Review \& Editing

Mengtao Xu: Resources and supervision

Hongzhuang Zhang: Resources, Writing- reviewing and editing, Supervision, Writing- Review \& Editing

Changyou Li: Conceived the presented idea

Guo Yao: Conceived the presented idea

Zhenyuan Li: Carried out the experiment

Huihui miao: Carried out the experiment

Chenyu Wang: Carried out the experiment

Yimin Zhang: Resources and supervision

Ethics approval

This chapter does not contain any studies with human participants or animals

performed by any of the authors.

\section{Consent to participate}

Not applicable. The article involves no studies on humans.

\section{Consent for publication}

\section{Reference}

All authors have read and agreed to the published version of the manuscript.

[1] Y. Altintas, A. Verl, C. Brecher, L. Uriarte, G. Pritschow, Erratum to "Machine tool feed drives" [CIRP Ann. Manuf. Technol. 60 (2011) 779-796], CIRP Annals, 61 (2012) 837.

[2] H. Ohta, E. Hayashi, Vibration of linear guideway type recirculating linear ball bearings, Journal of Sound and Vibration, 235 (2000) 847-861.

[3] T. Fujita, A. Matsubara, K. Yamazaki, Experimental characterization of disturbance force in a linear drive system with high-precision rolling guideways, International Journal of Machine Tools and Manufacture, 51 (2011) 104-111.

[4] R. Ramesh, M.A. Mannan, A.N. Poo, Error compensation in machine tools - a review: Part I: geometric, cutting-force induced and fixture-dependent errors, International Journal of Machine Tools and Manufacture, 40 (2000) 1235-1256. 
[5] X. Kong, W. Sun, B. Wang, B. Wen, Dynamic and stability analysis of the linear guide with time-varying, piecewise-nonlinear stiffness by multi-term incremental harmonic balance method, Journal of Sound and Vibration, 346 (2015) 265-283.

[6] P. Soleimanian, M. Mohammadpour, H. Ahmadian, Coupled tribo-dynamic modelling of linear guideways for high precision machining application, Proceedings of the Institution of Mechanical Engineers Part J-Journal of Engineering Tribology, 235 (2021) 711-737.

[7] P. Pawełko, S. Berczyński, Z. Grządziel, Modeling roller guides with preload, Archives of Civil and Mechanical Engineering, 14 (2014) 691-699.

[8] L. Yang, L. Wang, W. Zhao, Hybrid modeling and analysis of multidirectional variable stiffness of the linear rolling guideway under combined loads, Proceedings of the Institution of Mechanical Engineers Part C-Journal of Mechanical Engineering Science, 234 (2020) 2716-2727.

[9] Y. Sakai, T. Tanaka, Influence of lubricant on nonlinear vibration characteristics of linear rolling guideway, Tribology International, 144 (2020) 106124.

[10] C. Li, M. Xu, G. He, H. Zhang, Z. Liu, D. He, Y. Zhang, Time-dependent nonlinear dynamic model for linear guideway with crowning, Tribology International, 151 (2020) 106413.

[11] W. Wang, C. Li, Y. Zhou, H. Wang, Y. Zhang, Nonlinear dynamic analysis for machine tool table system mounted on linear guides, Nonlinear Dynamics, 94 (2018) 2033-2045.

[12] W. Wang, G. Shen, Y. Zhang, Z. Zhu, C. Li, H. Lu, Dynamic reliability analysis of mechanical system with wear and vibration failure modes, Mechanism and Machine Theory, 163 (2021) 104385.

[13] H. Ohta, K. Tanaka, Vertical Stiffnesses of Preloaded Linear Guideway Type Ball Bearings Incorporating the Flexibility of the Carriage and Rail, Journal of Tribology, 132 (2009).

[14] J.-P. Hung, Y.-L. Lai, C.-Y. Lin, T.-L. Lo, Modeling the machining stability of a vertical milling machine under the influence of the preloaded linear guide, International Journal of Machine Tools and Manufacture, 51 (2011) 731-739.

[15] H. Ohta, S. Kato, J. Matsumoto, K. Nakano, A Design of Crowning to Reduce Ball Passage Vibrations of a Linear Guideway Type Recirculating Linear Ball Bearing, Journal of Tribology, 127 (2005) 257-262.

[16] S.-W. Kwon, V.-C. Tong, S.-W. Hong, Five-degrees-of-freedom model for static analysis of linear roller bearing subjected to external loading, Proceedings of the Institution of Mechanical Engineers, Part C: Journal of Mechanical Engineering Science, 233 (2018) 2920-2938.

[17] W. Sun, X. Kong, B. Wang, X. Li, Statics modeling and analysis of linear rolling guideway considering rolling balls contact, Proceedings of the Institution of Mechanical Engineers, Part C: Journal of Mechanical Engineering Science, 229 (2014) 168-179.

[18] C. Yuan Lin, J. Pin Hung, T. Liang Lo, Effect of preload of linear guides on dynamic characteristics of a vertical column-spindle system, International Journal of Machine Tools and Manufacture, 50 (2010) 741-746. 
[19] H.T. Zou, B.L. Wang, Investigation of the contact stiffness variation of linear rolling guides due to the effects of friction and wear during operation, Tribology International, 92 (2015) 472-484.

[20] H. Ohta, Sound of Linear Guideway Type Recirculating Linear Ball Bearings, Journal of Tribology, 121 (1999) 678-685.

[21] W. Tao, Y. Zhong, H. Feng, Y. Wang, Model for wear prediction of roller linear guides, Wear, 305 (2013) 260-266.

[22] C. Liu, C. Zhao, B. Wen, Investigation on coupled vibration of machine tool table system with position deviations, The International Journal of Advanced Manufacturing Technology, 114 (2021) 2321-2337.

[23] P. Majda, Modeling of geometric errors of linear guideway and their influence on joint kinematic error in machine tools, Precision Engineering, 36 (2012) 369-378.

[24] J. Ma, D. Lu, W. Zhao, Assembly errors analysis of linear axis of CNC machine tool considering component deformation, The International Journal of Advanced Manufacturing Technology, 86 (2016) 281-289.

[25] G. Sun, G. He, D. Zhang, Y. Sang, X. Zhang, B. Ding, Effects of geometrical errors of guideways on the repeatability of positioning of linear axes of machine tools, The International Journal of Advanced Manufacturing Technology, 98 (2018) 2319-2333. [26] Y. Sun, D. Wang, H. Dong, R. Xue, S. Yu, Pre-deformation for assembly performance of machine centers, Chinese Journal of Mechanical Engineering, 27 (2014) 528-536.

[27] T. Onat Ekinci, J.R.R. Mayer, G.M. Cloutier, Investigation of accuracy of aerostatic guideways, International Journal of Machine Tools and Manufacture, 49 (2009) 478-487.

[28] T.A. Harris, M.N. Kotzalas, Rolling Bearing Analysis: Essential Concepts of Bearing Technology,5thed, Taylor and Francis, NewYork, 2007.

[29] J. Satterly, The Moments of Inertia of some Polyhedra, The Mathematical Gazette, 42 (2016) 11-13.

[30] J. Liu, Y. Shao, T.C. Lim, Vibration analysis of ball bearings with a localized defect applying piecewise response function, Mechanism and Machine Theory, 56 (2012) 156169.

[31] W. Wang, Y. Zhou, H. Wang, C. Li, Y. Zhang, Vibration analysis of a coupled feed system with nonlinear kinematic joints, Mechanism and Machine Theory, 134 (2019) 562-581.

[32] J.F. Archard, Contact and Rubbing of Flat Surfaces, Journal of Applied Physics, 24 (1953) 981-988.

[33] P.J. Torvik, On estimating system damping from frequency response bandwidths, Journal of Sound and Vibration, 330 (2011) 6088-6097.

[34] N.H. Chandra, A.S. Sekhar, Swept sine testing of rotor-bearing system for damping estimation, Journal of Sound and Vibration, 333 (2014) 604-620. 